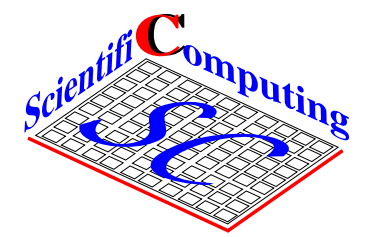

Galerkin Methods

for

\title{
Linear and Nonlinear Elliptic Stochastic Partial Differential Equations
}

\author{
Hermann G. Matthies, Andreas Keese \\ Institute of Scientific Computing \\ Technical University Braunschweig \\ Brunswick, Germany
}

Informatikbericht Nr.: 2003-08

July 2003

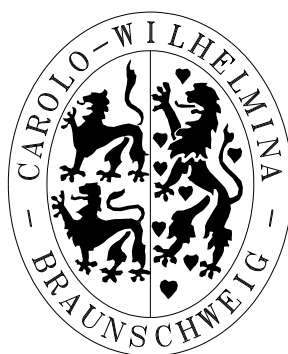




\title{
Galerkin Methods \\ for \\ Linear and Nonlinear Elliptic Stochastic Partial Differential Equations
}

\author{
Hermann G. Matthies, Andreas Keese \\ Department of Mathematics and Computer Science \\ Technical University Braunschweig \\ Brunswick, Germany
}

Informatikbericht Nr.: 2003-08

July 2003

Contributed to Computer Methods in Applied Mechanics and Engineering

\section{Location \\ Institute of Scientific Computing \\ Technische Universität Braunschweig \\ Hans-Sommer-Strasse 65 \\ D-38106 Braunschweig}

\section{Contact}

Phone: +49-(0)531-391-3000

Fax: +49-(0)531-391-3003

E-Mail: wire@tu-bs.de

HTTP: http://www.tu-bs.de/institute/WiR

\section{Copyright}

(c) Institut für Wissenschaftliches Rechnen

Technische Universität Braunschweig

\section{Postal Address}

Institut für Wissenschaftliches Rechnen

Technische Universtität Braunschweig

D-38092 Braunschweig

Germany 


\title{
Galerkin Methods \\ for \\ Linear and Nonlinear Elliptic Stochastic Partial Differential Equations
}

\author{
Hermann G. Matthies, Andreas Keese \\ Institute of Scientific Computing \\ Technical University Braunschweig \\ Brunswick, Germany \\ July 2003
}

\begin{abstract}
Stationary systems modelled by elliptic partial differential equationslinear as well as nonlinear-with stochastic coefficients (random fields) are considered. The mathematical setting as a variational problem, existence theorems, and possible discretisations-in particular with respect to the stochastic part-are given and investigated with regard to stability. Different and increasingly sophisticated computational approaches involving both Wiener's polynomial chaos as well as the Karhunen-Loève expansion are addressed in conjunction with stochastic Galerkin procedures, and stability within the Galerkin framework is established.

New and effective algorithms to compute the mean and covariance of the solution are proposed for various approaches. The similarities and differences with better known Monte Carlo methods are exhibited, as well as alternatives to integration in high-dimensional spaces. Hints are given regarding the numerical implementation and parallelisation. Numerical examples serve as illustration.
\end{abstract}

Keywords: linear and nonlinear elliptic stochastic partial differential equations, Galerkin methods, Karhunen-Loève expansion, Wiener's polynomial chaos, white noise analysis, sparse Smolyak quadrature, Monte Carlo methods, stochastic finite elements

AMS classification: 34F05 35R60 60H35 60H15 65N30 65C05 67S05 


\section{Contents}

1 Introduction 4

2 A Model Problem-the Linear Case $\quad 7$

2.1 Variational Description . . . . . . . . . . . . . 7

2.2 Stochastic Description . . . . . . . . . . . . . 9

2.2.1 The Karhunen-Loève Expansion . . . . . . . . . . . 10

2.2.2 Wiener's Polynomial Chaos Expansion . . . . . . . . . 12

2.3 Variational Requirements . . . . . . . . . . . . . . . . 15

3 Linear Systems with Stochastic Right Hand Side

-Additive Noise $\quad 15$

3.1 Mathematical Setting . . . . . . . . . . . . . . . 16

3.2 Discretisation and Numerical Approximation . . . . . . . . 18

3.2.1 Spatial Discretisation . . . . . . . . . . . . 19

3.2.2 Stochastic Discretisation . . . . . . . . . . . . 20

3.3 Computational Procedures . . . . . . . . . . . . . . 20

3.3.1 Monte Carlo Methods . . . . . . . . . . . . . . 21

3.3.2 Using the Polynomial Chaos Expansion . . . . . . . . . 22

3.3.3 Using the Karhunen-Loève Expansion . . . . . . . . . . . 23

3.3.4 Using the Karhunen-Loève Expansion of the Solution . . 24

3.3.5 Direct or Non-Intrusive Computation . . . . . . . . 27

3.4 The Solution as Filtered White Noise . . . . . . . . . . . . 28

4 Linear Systems with Stochastic Operator

-Multiplicative Noise $\quad 29$

4.1 Mathematical Formulation . . . . . . . . . . . . . . . . . . . 29

4.2 Discretisation and Numerical Approximation . . . . . . . . 30

4.3 Computational Procedures . . . . . . . . . . . . . . 31

4.3.1 Monte Carlo Methods-Continued . . . . . . . . . . 31

4.3.2 Can we use the Polynomial Chaos Expansion? . . . . . . 32

4.3.3 Using the Karhunen-Loève Expansion of the Matrix . . . 34

4.3.4 Using the Karhunen-Loève Expansion of the Solution . . 39

4.3.5 Direct or Non-Intrusive Computation-Continued . . . . 43

5 The Nonlinear Case $\quad 43$

5.1 Mathematical Formulation . . . . . . . . . . . . . . . . 44

5.2 Discretisation and Numerical Approximation . . . . . . . . . . 45

5.3 Computational Procedures . . . . . . . . . . . . . 46

5.3.1 Monte Carlo Methods-Encore . . . . . . . . . . . . . 46 
5.3.2 Using the Polynomial Chaos Expansion . . . . . . . . . 46

5.3.3 Direct or Non-Intrusive Computation-Finale . . . . . . 49

5.3.4 High-Dimensional Integration . . . . . . . . . . 50

6 Conclusions

53 


\section{Introduction}

In many applications the values of the parameters of the problem are not exactly known. We may take the simulation of groundwater flow as an example. Knowledge about the conductivity of the soil, the magnitude of source-terms, or about the in- and out-flow boundary conditions is often very uncertain. These uncertainties inherent in the model result in uncertainties in the results of numerical simulations.

Stochastic methods are one way to model these uncertainties, and in our case we are concerned with spatially varying randomness, and shall model this by random fields $[1,72,11]$. If the physical system is described by a partial differential equation (PDE), then the combination with the stochastic model results in a stochastic partial differential equation (SPDE). The solution of the SPDE is again a random field, describing both the expected response and quantifying its uncertainty.

SPDEs can be interpreted mathematically in several ways. At the moment we concentrate on randomness in space. If evolution with stochastic input has to be considered, one may combine the techniques described here with the already well established methods in that field [38]; for theoretical results, e.g. see [50].

We shall distinguish - as in the case of stochastic ordinary differential equations (SDEs) - between additive and multiplicative noise. As is well known from SDES, in the case of multiplicative noise one has to be more careful. A similar problem occurs here. Additive noise-particularly for linear problems-is much simpler to deal with. The random fields may be generalised to stochastic distributions, and with multiplicative noise the product of random coefficient field and solution may have no meaning in this case. As with SDEs, it is a modelling decision how this is resolved.

Additive noise corresponds to the case where the right hand side - the loading or the solution independent source terms - is random, whereas when the operator is random, we have multiplicative noise. In the first case it is the external influences which are random, in the latter it is the system under consideration itself.

A theory of SPDEs where products between random fields are interpreted as Wick products was developed in [27]. This allows highly irregular random fields as coefficients, and obtains the solution as a stochastic Kondratiev distribution. Its main shortcoming is that-e.g. for linear problems-higher statistical moments of system parameters do not influence the mean of the solution, a contradiction to the results of homogenisation theory. Another problem is the required existence of strong solutions [27] to the PDE. These may be relaxed by a variational formulation $[71,46,70]$, but nonetheless the Wick product seems not to be the right model for the problems that we aim at.

For products interpreted in the usual sense, stronger regularity is required for 
the coefficient random random fields [9], still allowing the stochastic part of the solution to be a Kondratiev distribution (see [27]).

In the numerical framework, the stochastic regularity of the solution determines the convergence rate of numerical approximations, and a variational theory for this was earlier devised in [71, 8] and extended in [70]. Further results in a more restricted setting can be found in [14, 4, 6, 7, 65].

The ultimate goal in the solution of SPDEs is usually the computation of response statistics, i.e. functionals of the solution. Monte Carlo (MC) methods can be used directly for this, but they require a high computational effort [10]. Variance reduction techniques are employed to reduce this somewhat. Quasi Monte Carlo (QMC) methods $[10,51]$ may reduce the computational effort considerably without requiring much regularity. But often we have high regularity in the stochastic variables, and this is not exploited by QMC methods. We propose sparse (Smolyak) quadrature methods as an efficient alternative. These have first been described in [67] and have found increasing attention in recent years, e.g. $[52,60,19]$.

Alternatives to Monte Carlo (e.g. [56, 17]) methods, which first compute the solution, and then the required statistic, have been developed in the field of stochastic mechanics - cf. [40, 41], for example perturbation methods, e.g. [37], methods based on Neumann-series, e.g. [22, 4], or the spectral stochastic finite element-method (SSFEM) [22]. The latter expands the random fields in eigenfunctions of their covariance kernels, and obtains the solution by a Galerkin method in a space of stochastic ansatz functions. More information, references and reviews on stochastic finite elements can be found in [45, 63, 69, 31]. A somewhat specialised field is the area of structural reliability, e.g. see [16, 26].

Following [22], stochastic Galerkin methods have been applied to various linear problems, e.g. [20, 21, 57, 49, 32, 18, 30, 74, 75]. Recently, nonlinear problems with stochastic loads have been tackled, e.g. [75], and some first results of both a theoretical and numerical nature for nonlinear stochastic operators are in $[33,36]$.

These Galerkin methods allow us to have an explicit functional relationship between the independent random variables and the solution-and this is contrast with usual Monte Carlo approaches, so that subsequent evaluations of functionals-statistics like the mean, covariance, or probabilities of exceedenceare very cheap. This may be seen as a way to systematically calculate more and more accurate "response surfaces" [39].

The plan of the paper is as follows: In section 2 we introduce a model problem, a prototype model of stationary groundwater flow, cast in a variational formulation, to serve as a guide for the stochastic problem in section 2.1. The stochastic descriptions in section 2.2 involves two expansions, the Karhunen-Loève expansion in the spatial variables, and Wiener's polynomial chaos expansion in the 
stochastic variables. Although we do not assume Gaussian random fields, we model them as functions of Gaussian random fields, so that in the end everything is expressed in terms of Gaussian variables, which are our basic building blocks. In section 2.3 we match the stochastic description against the requirements which come from the variational theory of partial differential equations in order to have a well-posed problem in the sense of Hadamard.

We start with the simplest case in section 3, that is a linear partial differential equation where just the right-hand-side or source term is a random field, something which may be called additive noise. The mathematical formulation is extended to accommodate the stochastic variables and their discretisation. As the best known approach the Monte Carlo method and some variants are reviewed first, so that the Galerkin approach may be put into perspective. The computational paths for Galerkin methods may be graded according to their degree of sophistication, and we show three variants.

We propose a new method to compute the Karhunen-Loève basis of the solution through the resolution of a generalised eigenvalue problem. We show how Monte Carlo like ideas may be used in conjunction with the polynomial chaos expansion.

The next in difficulty is still the linear partial differential equation, but with a random field in the operator, which is addressed in section 4 . This may be termed as multiplicative noise. The mathematical description is adapted to this situation, and we show how the previous results with just stochastic source terms may be used here. The computational approaches are analogous to those in section 3 . We show the stability of the approximation in conjunction with Galerkin methods without any additional assumptions on the stochastic coefficient.

The Galerkin methods lead to equations with tensor product structure, and this may be used to great advantage in the numerical solution.

The Karhunen-Loève expansion of the solution is the best basis, and we propose a new approach to compute it through a Neumann series. The KarhunenLoève expansion may be used to great advantage in reducing the spatial size of the system.

In section 5 we describe stochastic Galerkin methods for general nonlinear elliptic boundary value problems, starting as before with the mathematical setting. We give new existence theorems, and again address the discretisation, following the previous pattern as much as possible. We indicate how to solve the resulting large nonlinear system by a preconditioned $B F G S$-scheme.

Alternatively, we obtain the solution by projecting orthogonally onto the stochastic ansatz-space, using sparse Smolyak quadrature.

We illustrate the proposed methods by numerical examples and show some numerical experiments regarding computational alternatives.

Finally we conclude with an outlook and some open problems. 


\section{A Model Problem-the Linear Case}

In order to have something concrete to work with, we take a simple model problem, which nevertheless exhibits all the salient features which come with a stochastic system.

\subsection{Variational Description}

To be a bit more specific, we assume that we are dealing with a stationary diffusion equation

$$
\begin{aligned}
-\nabla^{T}(\kappa(x) \nabla u(x)) & =f(x) \quad \text { for } \quad x \in R, \\
u(x) & =0 \text { for } \quad x \in \partial R .
\end{aligned}
$$

Here $R$ is some bounded, admissible region in $\mathbb{R}^{d}, \partial R$ its boundary, and we have assumed homogenous essential boundary conditions for the sake of simplicity. The solution $u$ may be a concentration, or temperature; $\kappa$ is the appropriate conductivity, and the right hand side (RHS) $f$ are sources or sinks in the region $R$.

This may be taken as a very simple description of groundwater flow, which is the application we have in mind, see the graphical description of the model problem in Fig. 1. This is a two-dimensional L-shaped domain (the flow is averaged over the depth, because the horizontal extent is much larger then in the vertical), and the RHS and boundary conditions are indicated. In that case $u$ is the hydraulic head, and $\kappa$ the hydraulic conductivity. We shall later allow that $\kappa$ depends on the solution $u$, and hence the problem may be nonlinear, but here we deal with the linear case first.

We first put the deterministic problem into a variational formulation, which we use later on also for the stochastic case. This is completely standard, and is only included here for reference, and because we want to use it to build the stochastic variational statement with it. We set for the admissible space of solutions $V=$ $\stackrel{\circ}{H}^{1}(R)$, the dual space of which is $V^{*}=H^{-1}(R)$. Assuming that $\kappa \in L_{\infty}(R)$ and $f \in V^{*}$, we may formulate Eq.(1) as a variational problem on $V$ : Find $u \in V$ such that for all $v \in V$ :

$$
\mathrm{b}(v, u):=\int_{R}(\nabla v(x))^{T} \kappa(x) \nabla u(x) d x=\langle f, v\rangle:=\int_{R} f(x) v(x) d x .
$$

Proposition 1. If $\kappa(x) \geq \kappa_{-}>0$ a.e. in $R$, this problem has a unique solution depending continuously on $f$ and on $\kappa$.

Proof. This is a simple consequence of the Lax-Milgram lemma, as the bilinear form $\mathrm{b}(\cdot, \cdot)$ is both continuous and coercive- $\mathrm{b}(u, u) \geq C\|u\|^{2}-$ on $V$. The 


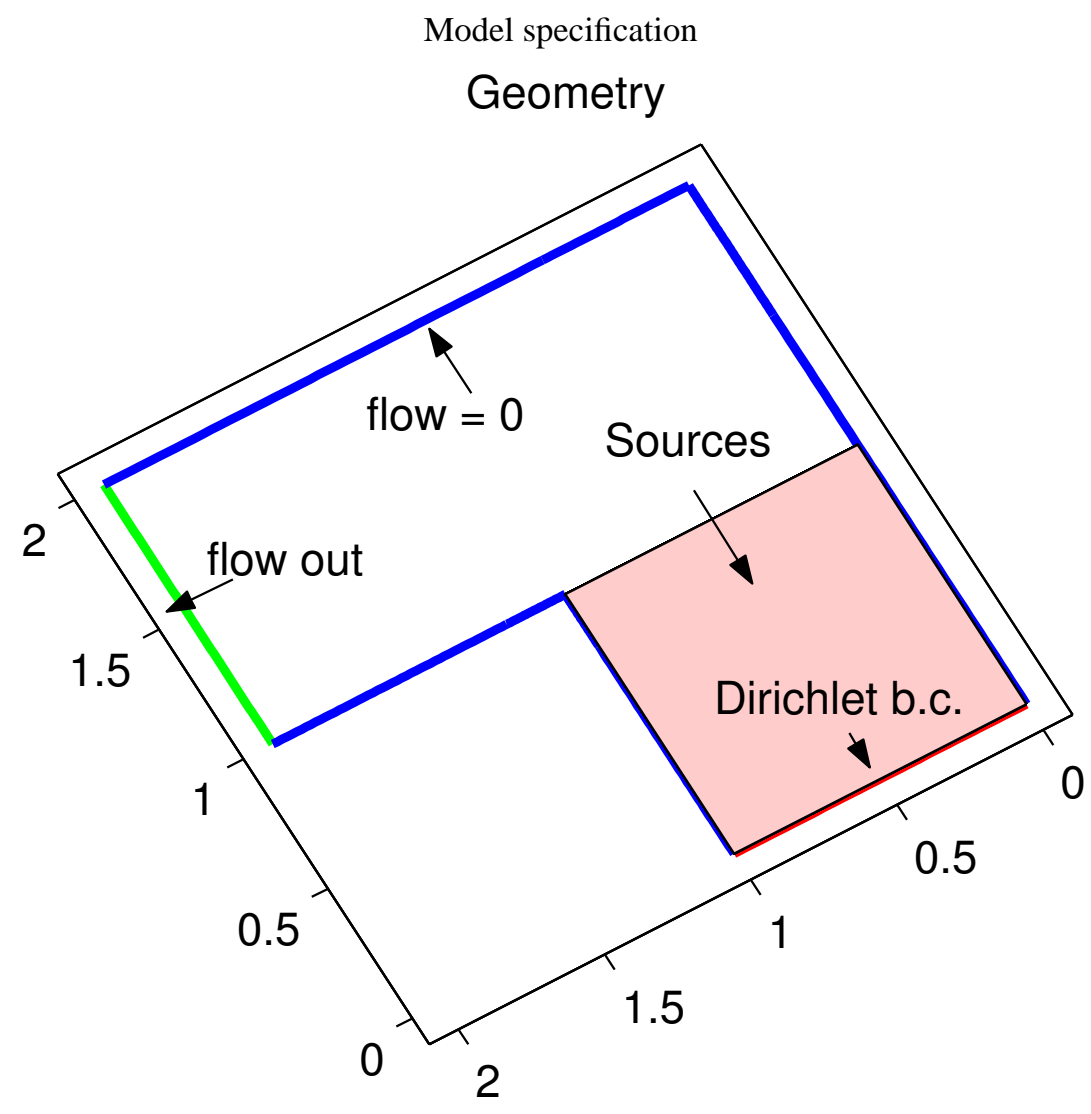

Figure 1: Model Problem and Geometry.

continuous dependence on $\kappa$ in the $L_{\infty}$-norm may be seen as a consequence of the first Strang lemma (cf. [12, 68]).

A rewording of this result is the following corollary:

Corollary 1. As is well known, the bilinear form thus defines a linear, continuous, self-adjoint and positive definite operator $\mathrm{B}: V \rightarrow V^{*}$, which has a continuous inverse. The operator $\mathrm{B}$ and its inverse depend continously on $\kappa$. The PDE may be written in this interpretation as $\mathrm{B} u=f$.

The variational problem therefore satisfies all of Hadamard's requirements for a well-posed problem: it has a solution, the solution is unique, and it depends continuously on the data. We would like to take this as our 'leitmotiv' when we approach the stochastic case, we want to still have well-posed problems. Also, as 
neither the deterministic nor the stochastic problem can usually be solved analytically, we have to employ numerical approximations; and these we would like to be well-posed as well.

\subsection{Stochastic Description}

Both the external influences - the source terms or the RHS $f$ - and the system, represented by the conductivity $\kappa$, may be uncertain. The uncertainty in the hydraulic conductivity for example may be modelled by defining $\kappa(x)$ for each $x \in R$ as a random variable $\kappa(x): \Omega \rightarrow \mathbb{R}$ on a suitable probability space $(\Omega, \mathcal{B}, \Gamma)$. Here $\Omega$ is the set of elementary events, $\mathcal{B}$ a $\sigma$-algebra and $\Gamma$ a probability measure. As a consequence, $\kappa: R \times \Omega \rightarrow \mathbb{R}$ is a random field $[1,72,11]$, and one may identify $\Omega$ with the set of "all possible soil properties", i.e. with the space of all realisations $\kappa(\cdot, \omega): R \rightarrow \mathbb{R}, \omega \in \Omega$, i.e. $\kappa(x, \omega)=\omega(x)$-a possible realisation is shown in Fig. 2. As this space is usually infinite dimensional, the probability space is in some sense infinite dimensional as well.

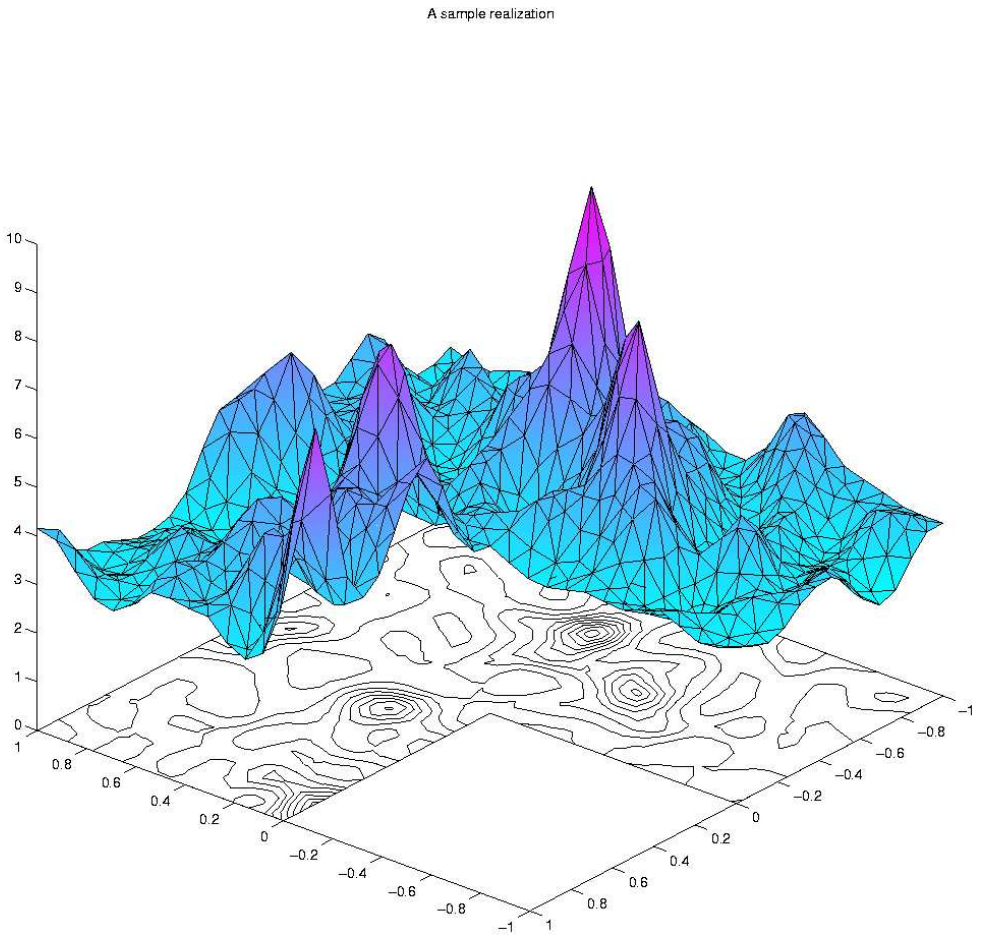

Figure 2: Realisation of $\kappa$.

It is of course also possible that the boundary conditions are uncertain, or even 
the shape or size of the region $R$ [5]. Here we are content with just looking at the effect of an uncertain conductivity and RHS.

The probability space can be defined by the joint statistic of all possible combinations $\kappa\left(x_{1}\right), \ldots, \kappa\left(x_{m}\right), m \in \mathbb{N}, x_{1}, \ldots, x_{m} \in R$ (all finite-dimensional distributions), see e.g. [1] for details. Often, only second order statistics (mean and covariance) and marginal distributions - the distribution of the random variable $\omega \mapsto \kappa\left(x_{0}, \omega\right)$ for a particular point $x_{0}$-are known from measurements. For later reference, we note that such a random field may be described by a transformation

$$
\kappa(x, \omega)=\phi(x, \gamma(x, \omega)), \quad x \in R, \omega \in \Omega,
$$

of a Gaussian random field $\gamma(x, \omega)$, defined by its mean

$$
\left.\bar{\gamma}(x):=\mathbf{E}(\gamma(x, \cdot))):=\int_{\Omega} \gamma(x, \omega)\right) \Gamma(d \omega)
$$

and its covariance

$$
\left.\left.C_{\gamma}(x, y):=\mathbf{E}((\gamma(x, \cdot)-\bar{\gamma}(x))(\gamma(y, \cdot)-\bar{\gamma}(y))):=\int_{\Omega} \gamma(x, \omega)\right) \gamma(y, \omega)\right) \Gamma(d \omega) .
$$

Here $\mathbf{E}(\cdot)$ is the expectation operator, the averaging over $\Omega$. Without loss of generality we may choose $\gamma(x, \omega)$ to have zero mean $(\bar{\gamma}(x)=0)$ and unit variance $\left(C_{\gamma}(x, x)=1\right)$.

It is well-known that a normally distributed, zero mean and unit variance Gaussian random variable $\mathcal{N}(0,1)$ can be mapped to a random variable with distribution-function $F_{\kappa}$ by the transformation $F_{\kappa}^{-1}(\operatorname{erf}(\mathcal{N}(0,1)))$, where erf is the Gaussian distribution-function. In addition, the combination of $\phi$ and $C_{\gamma}(x, y)$ may be chosen such that $\kappa(x, \omega)$ satisfies given second order statistics (cf. [62]). Given the average $\bar{\kappa}(x):=\mathbf{E}(\kappa(x, \cdot))$, we decompose

$$
\kappa(x, \omega)=\bar{\kappa}(x)+\tilde{\kappa}(x, \omega)
$$

(and later also all other stochastic processes) into the mean and the zero mean fluctuating part $\tilde{\kappa}(x, \omega)$; the correlation function of $\kappa$ is then given by

$$
C_{\kappa}(x, y):=\mathbf{E}((\kappa(x, \cdot)-\bar{\kappa}(x))(\kappa(y, \cdot)-\bar{\kappa}(y)))=\mathbf{E}(\tilde{\kappa}(x, \cdot) \tilde{\kappa}(y, \cdot)) .
$$

\subsubsection{The Karhunen-Loève Expansion}

One prime possibility to have a computationally feasible representation of the random field is the Karhunen-Loève (KL) expansion. Here we take the conductivity $\kappa(x, \omega)$ as an example. But everything we say applies to any other stochastic field, e.g. the RHS $f(x, \omega)$ and the Gaussian field $\gamma(x, \omega)$ in Eq.(3) (cf. [66]). 
The spatial KL-expansion functions $\kappa_{\jmath}(x)$ are the eigenfunctions of the Fredholm integral equation with the covariance function as the integral kernel:

$$
\int_{R} C_{\kappa}(x, y) \kappa_{\jmath}(y) d y=\lambda_{\jmath} \kappa_{\jmath}(x)
$$

and the eigenvalues $\lambda_{\jmath}$ tell us how much stochastic variance is carried by that particular eigenfunction. As Eq.(4) is a compact, symmetric, and positive semidefinite operator on $L_{2}(R)$, the spectrum is real, and consists entirely of eigenvalues $\lambda_{\jmath}>0$, which may be ordered in a descending order with possible accumulation point zero, see $[13,61]$. For our example problem, two such eigenfunction

Eigenfunction No. 1

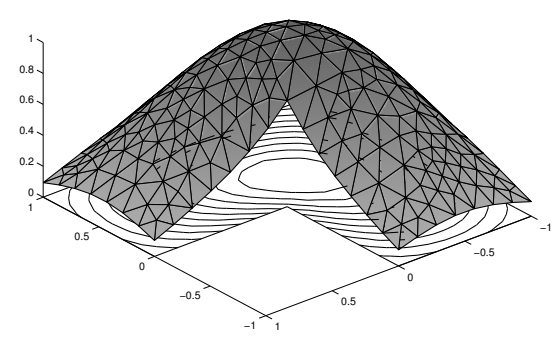

Eigenfunction No. 15

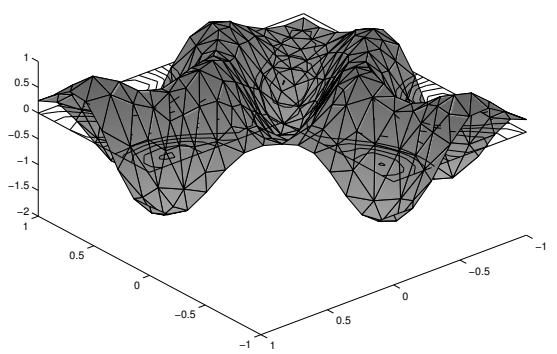

Figure 3: KL-Eigenfunctions Nos. 1 and 15.

are shown in Fig. 3. These eigenfunctions form a complete orthonormal set in $L_{2}(R)$, and the covariance kernel may be expanded in the series

$$
C_{\kappa}(x, y)=\sum_{\jmath=1}^{\infty} \lambda_{\jmath} \kappa_{\jmath}(x) \kappa_{\jmath}(y) .
$$

In case the covariance function is continuous, Mercer's theorem (cf. [13, 61]) tells us that this series converges not only in $L_{2}(R \times R)$, but uniformly. Usually it is not possible to solve Eq.(4) analytically, and it has to be done numerically. This is a standard numerical problem, and we will not further dwell on it, but instead refer to e.g. [2].

The KL-expansion of the stochastic process $\kappa(x, \omega)$ may now be stated as (cf. [42]):

$$
\kappa(x, \omega)=\sum_{\jmath=1}^{\infty} \sqrt{\lambda_{\jmath}} \xi_{\jmath}(\omega) \kappa_{\jmath}(x) .
$$


This converges in $L_{2}(R \times \Omega)$. It is a generalisation of the well-known Fourier expansion of stochastic processes on the real line, when the covariance function is homogenous, i.e. depends only on the difference $|x-y|$. The random variables $\xi_{\jmath}(\omega)$ are the 'Fourier' coefficients, have zero mean and unit variance, and are given for positive eigenvalues by

$$
\xi_{\jmath}(\omega):=\left(\lambda_{\jmath}\right)^{-1 / 2} \int_{R} \kappa_{\jmath}(x) \kappa(x, \omega) d x .
$$

As the eigenfunctions $\left\{\kappa_{\jmath}(x)\right\}$ are orthonormal

$$
\left\langle\kappa_{\jmath}(\cdot), \kappa_{\ell}(\cdot)\right\rangle=\int_{R} \kappa_{\jmath}(x) \kappa_{\ell}(x) d x=\delta_{\jmath \ell}
$$

so are the coefficient random variables $\left\{\xi_{\jmath}(\omega)\right\}$, but in $L_{2}(\Omega)$ :

$$
\left\langle\xi_{\jmath}(\cdot) \mid \xi_{\ell}(\cdot)\right\rangle_{L_{2}(\Omega)}:=\mathbf{E}\left(\xi_{\jmath}(\cdot) \xi_{\ell}(\cdot)\right)=\int_{\Omega} \xi_{\jmath}(\omega) \xi_{\ell}(\omega) \Gamma(d \omega)=\delta_{\jmath \ell}
$$

Stochastically speaking this means that they are uncorrelated, but in general not independent.

Smoothness of the covariance function-mirrored by the decay of its spectrum-will give better approximation properties for the KL-expansion of the stochastic process [6].

\subsubsection{Wiener's Polynomial Chaos Expansion}

Later we will want to integrate numerically over $\Omega$. In order to facilitate this, it is better to use independent random variables (then Fubini's theorem can be applied), and best is to use independent identically distributed (iid) random variables. There is one type of random variable, where being uncorrelated implies independence - this is the defining property of Gaussian random variables. The Gaussian variables form a closed subspace $G$ in $L_{2}(\Omega)$, a Gaussian Hilbert space $[28,27,43]$. As Gaussian variables possess moments of any order, they also form a (graded) algebra with unit w.r.t. multiplication in $L_{2}(\Omega)$. Norbert Wiener [73] has shown that any random variable $\xi \in L_{2}(\Omega)$ may be expanded in polynomials of Gaussian variables (the polynomials are in the algebra), and in particular in multi-dimensional polynomials orthogonal w.r.t. Gaussian measure, the Hermite polynomials, see $[28,27,43]$. So the algebra is dense in $L_{2}(\Omega)$. At the risk of abusing notation, we shall call these Gaussian variables again $\omega \in \Omega$, and an orthonormal - uncorrelated and independent—basis in $G$ can be compared to "Cartesian coordinates" on $\Omega$. The Hermite polynomials have these Gaussian 
variables $\omega$ from $G \subset L_{2}(\Omega)$ as their argument. The measure to be used in conjunction with them is of course a Gaussian measure, and-repeating the abuse of notation-it will again be called $\Gamma$.

The multi-variate Hermite polynomials are defined as follows: Let the multiindices $\alpha=\left(\alpha_{1}, \ldots, \alpha_{\jmath}, \ldots\right) \in(\mathbb{N})_{c}^{\mathbb{N}}=: \mathcal{J}$ be sequences of non-negative integers, only finitely many of which are non-zero, and let $h_{k}(x)$ denote the usual onedimensional Hermite polynomial of order $k \in \mathbb{N}_{0}$. Then define

$$
H_{\alpha}(\omega):=(\alpha !)^{-1 / 2} \prod_{\jmath=1}^{\infty} h_{\alpha_{\jmath}}\left(\omega_{\jmath}\right),
$$

where $\alpha !:=\prod_{\jmath=1}^{\infty}\left(\alpha_{\jmath} !\right)$; for later reference let us also define $|\alpha|:=\sum_{\jmath=1}^{\infty} \alpha_{\jmath}$. As $h_{0} \equiv 1$, and only finitely many $\alpha_{\jmath} \neq 0$, all expressions are well defined. Two examples of such polynomials may be seen in Fig. 4 . The factor $(\alpha !)^{-1 / 2}$ makes

$$
H_{(2,3)}
$$

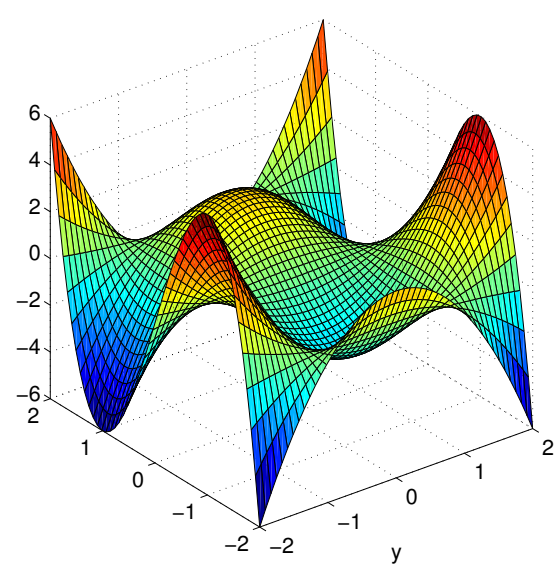

$H_{(3,3)}$

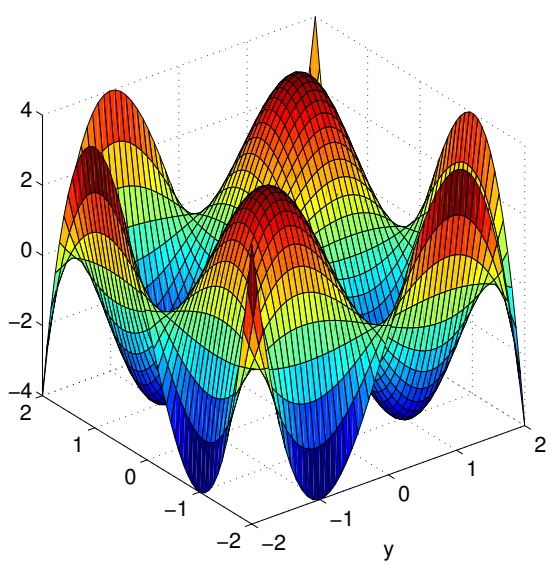

Figure 4: Hermite Polynomials $H_{(2,3)}$ and $H_{(3,3)}$.

these functions orthonormal in $L_{2}(\Omega)$ with a standard Gaussian measure $\Gamma(d \omega)$,

$$
\left\langle H_{\alpha} \mid H_{\beta}\right\rangle_{L_{2}(\Omega)}:=\mathbf{E}\left(H_{\alpha} H_{\beta}\right)=\int_{\Omega} H_{\alpha}(\omega) H_{\beta}(\omega) \Gamma(d \omega)=\delta_{\alpha \beta} .
$$

Each random variable $\xi$ with finite variance $-\xi \in L_{2}(\Omega)$ - can be expanded into a series of these polynomials, convergent in $L_{2}(\Omega)$ :

$$
\xi(\omega)=\sum_{\alpha \in \mathcal{J}} c^{(\alpha)} H_{\alpha}(\omega)
$$


where $\omega=\left(\omega_{1}, \ldots, \omega_{\jmath}, \ldots\right)$.

As $\kappa(x, \omega)$ is a.e. in $x$ such a random variable, we can use this here too:

$$
\kappa(x, \omega)=\sum_{\alpha \in \mathcal{J}} \kappa^{(\alpha)}(x) H_{\alpha}(\boldsymbol{\omega}),
$$

where now of course the coefficients $\kappa^{(\alpha)}(x)$ are dependent on the location $x$, i.e. they are spatial functions.

In the KL-expansion Eq.(6), each $\xi_{\jmath}$ can be expanded in such polynomials:

$$
\xi_{\jmath}(\omega)=\sum_{\alpha \in \mathcal{J}} c_{\jmath}^{(\alpha)} H_{\alpha}(\boldsymbol{\omega}) .
$$

The random variables $\left\{\omega_{j}\right\} \in \mathcal{N}(0,1)$ are uncorrelated, and as they are Gaussian, they are also independent. Now finally we have a description of the stochastic process as a function of a countable collection of iid random variables, a property which is important for the subsequent computations.

Altogether we then have

$$
\kappa(x, \omega)=\sum_{\jmath=1}^{\infty} \sum_{\alpha \in \mathcal{J}} \sqrt{\lambda_{\jmath}} c_{\jmath}^{(\alpha)} H_{\alpha}(\boldsymbol{\omega}) \kappa_{\jmath}(x) ;
$$

and obviously the factor $\sqrt{\lambda_{\jmath}}$ could be absorbed in the coefficient $c_{\jmath}^{(\alpha)}$. A reali-

Realisation with 20 KL-terms

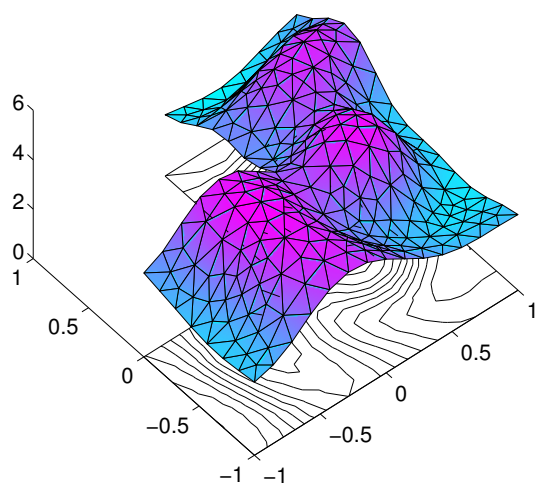

Realisation with $50 \mathrm{KL}$-terms

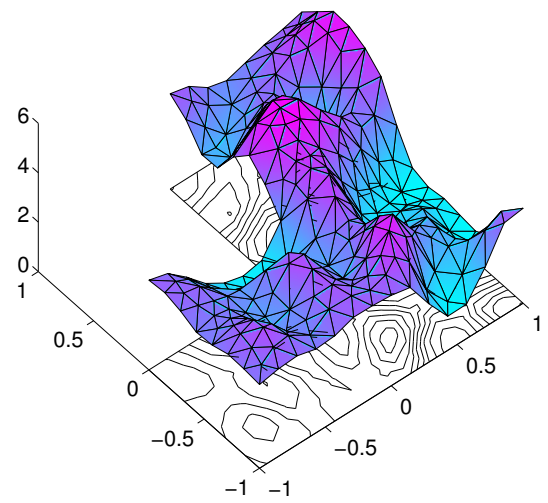

Figure 5: Realisations of $\kappa$ with 20 and 50 KL-Terms.

sation of $\kappa(x, \omega)$ generated in this way with 20 and with 50 KL-eigenfunctions is shown in Fig. 5. 


\subsection{Variational Requirements}

For a physically and mathematically well-defined model, the conductivity should be bounded from above and below,

$$
0<\kappa_{-}<\kappa(x, \omega)<\kappa_{+}<\infty \text {, a.e. }
$$

This is guaranteed by a suitable choice of $\phi$ in Eq. (3). In our computations we assign to $\kappa(x)$ a $\beta(1 / 2,1 / 2)$-distribution (the probability distribution has the shape of half a sine-wave). The transformation is

$$
\kappa(x, \omega)=c_{1}(x)+c_{2}(x) \arccos (\operatorname{erf}(\gamma(x, \omega))) .
$$

Uncertainties in the other parameters may be modelled similarly, and a combination with Eq. (1) yields the stochastic PDE for $\Gamma$-almost all $\omega \in \Omega$ :

$$
\begin{aligned}
-\nabla^{T}(\kappa(x, \omega) \nabla u(x, \omega)) & =f(x, \omega) \text { for } x \in R, \\
u(x, \omega) & =0 \text { for } x \in \partial R,
\end{aligned}
$$

where we have similarly to the conductivity also assumed that the source $f(x, \omega)$ may be random.

The hydraulic head, the solution $u(x, \omega)$ of Eq.(14), is also a random field, and our goal is usually to compute response statistics, e.g. the expected hydraulic head $\bar{u}(x)=\mathbf{E}(u(x, \cdot))$, the covariance $C_{u}(x, y)=\mathbf{E}((\tilde{u}(x, \cdot))(\tilde{u}(y, \cdot)))$, or the probability that the hydraulic head exceeds some threshold, $p_{u}(x)=\operatorname{Prob}\{u(x, \cdot)>$ $\left.u_{0}\right\}=\mathbf{E}\left(\chi_{\left(u_{0}, \infty\right)}(u(x, \cdot))\right)$. All these expressions $\Psi_{u}(x)$ are functionals of the solution $u(x, \omega)$, and in particular are integrals with respect to the measure $\Gamma(d \omega)$,

$$
\Psi_{u}(x)=\mathbf{E}(\Psi(x, \cdot, u(x, \cdot)))=\int_{\Omega} \Psi(x, \omega, u(x, \omega)) \Gamma(d \omega) .
$$

The numerical evaluation of such statistics requires a discretisation of Eq.(14) in space and in the stochastic "dimension", and both discretisations may be performed independently of each other. Almost any technique may be used for the spatial discretisation, and we use finite elements in section 3.2.1.

Once the problem is discretised in space, a stochastic discretisation has to be chosen, as we have to approximate Eq.(14) in a finite number of independent random variables. This will be done in section 3.2.2.

\section{Linear Systems with Stochastic Right Hand Side -Additive Noise}

We want to introduce the stochastic problems one difficulty at the time. For this purpose the consideration of a deterministic system with a stochastic right hand 
side seems to be a first good step $[14,18,65]$. This kind of situation is the simplest of the stochastic problems, and it allows us to introduce our approach at an easy pace. It also is most similar to what is common knowledge about linear timeinvariant dynamical systems with random (time varying) inputs. There we can use the Fourier transform, and then it is essentially the transfer function of the system - or rather the square of the transfer function-which maps the spectrum of the input process onto the spectrum of the output. A similar situation will be observed here, but the Fourier transform has to be generalised to the KarhunenLoève expansion.

\subsection{Mathematical Setting}

We assume that $f$ at each $x \in R$ is a random variable, i.e. $f \in H^{-1}\left(R,(S)^{*}\right)$, where $(S)$ is an appropriate separable Hilbert space of random variables. For the sake of simplicity we shall take here $(S)=L_{2}(\Omega)$, but other choices are possible $[27,8,70]$. As $H^{-1}\left(R,(S)^{*}\right)$ is isomorphic to $H^{-1}(R) \otimes(S)^{*}, f$ may be represented as

$$
f(x, \omega)=\sum_{k, \ell} f_{k}^{(\ell)} u_{k}(x) \varphi_{\ell}(\omega), \quad(x, \omega) \in R \times \Omega,
$$

where $\left\{u_{k}\right\}$ is a basis in $H^{-1}(R)=V^{*}$, and $\left\{\varphi_{\ell}\right\}$ a basis in $(S)^{*}$, and $V$ and $V^{*}$ are as before in section 2.1. We also need here that the gradient $\nabla: V \rightarrow Q$ is continuous, where $Q=L_{2}\left(R, \mathbb{R}^{d}\right)$.

Definition 1. We shall look for solutions in $V \otimes(S)$, and define the operator

$$
\nabla_{\omega}:=\nabla \otimes I d: V \otimes(S) \rightarrow Q \otimes(S)
$$

by looking how it acts on a single product term $u(x) \varphi(\omega)$, where $u \in V$ and $\varphi \in(S)$ :

$$
\nabla_{\omega}: u(x) \varphi(\omega) \mapsto(\nabla u(x)) \varphi(\omega) .
$$

This may then extended by linearity and continuity to the whole space.

Let us now formulate the stochastic variational problem: Find $u \in V \otimes(S)$ such that for all $v \in V \otimes(S)$ :

$$
\begin{aligned}
& \mathbf{b}(v, u):=\int_{\Omega} \int_{R}\left(\nabla_{\omega} v(x, \omega)\right)^{T} \kappa(x)\left(\nabla_{\omega} u(x, \omega)\right) d x \Gamma(d \omega)= \\
&\langle\langle f, v\rangle\rangle:=\int_{\Omega} \int_{R} f(x, \omega) v(x, \omega) d x \Gamma(d \omega) .
\end{aligned}
$$

We assume the same conditions as before for the deterministic problem, and then it is not hard to see that we have $[71,8,70,14]$ : 
Proposition 2. The variational problem Eq.(17) has a unique solution for all $f \in$ $(V \otimes(S))^{*}$, depending continously on $f$ and on $\kappa$.

Proof. Using the representation $f(x, \omega)=\sum_{k, \ell} f_{k}^{(\ell)} u_{k}(x) \varphi_{\ell}(\omega)$, it is easy to see that $\langle\langle f, v\rangle\rangle$ is continuous on products $u(x) \varphi(\omega) \in V \otimes(S)$, and as the span of these is dense, $\langle\langle f, v\rangle\rangle \leq C\|v\| \quad \forall v \in V \otimes(S)$.

In the same way we may show the continuity and coerciveness of $\mathbf{b}$. Due to the Lax-Milgram lemma, there is a unique solution $u \in V \otimes(S)$, depending linearly and continuously on $f$. The continuous dependence on $\kappa$ follows again from the first Strang lemma (cf. [68, 12]), see also [4, 6, 7].

As before, we may reword this in the language of operators in the following corollary:

Corollary 2. The bilinear form in Eq.(17) defines a linear, continuous, selfadjoint and positive definite operator $\mathbf{B}: V \otimes(S) \rightarrow(V \otimes(S))^{*}=V^{*} \otimes(S)^{*}$, which has a continuous inverse. Both the operator and its inverse depend continuously an $\kappa$. The SPDE may be written with this interpretation as $\mathbf{B} u=f$.

With this, we have carried over Hadamard's requirements for a well-posed problem to partial differential equations with a stochastic RHS.

As the solution $u(x, \omega)$ is now also a stochastic field, we may be interested in its statistics, e.g. its mean

$$
\bar{u}(x):=\mathbf{E}(u(x, \cdot)):=\int_{\Omega} u(x, \omega) \Gamma(d \omega),
$$

or its covariance

$$
C_{u}(x, y):=\mathbf{E}((u(x, \cdot)-\bar{u}(x))((u(y, \cdot)-\bar{u}(y))) .
$$

For the mean the following result holds (cf. $[3,14,65])$ :

Proposition 3. For the linear problem with stochastic RHS, the mean of the solution Eq.(18) satisfies the same differential equation Eq.(1) as the solution of the deterministic variational problem Eq.(2), with the mean of the stochastic RHS $\bar{f}(x):=\mathbf{E}(f(x, \cdot))$ as source term.

Proof. Letting $v(x, \omega)=v(x) \varphi(\omega)$ with $\varphi(\omega) \equiv 1$ in Eq.(17), we see that by taking expectations on both sides

$$
\begin{aligned}
\mathbf{b}(v, u)=\mathbf{E}(\mathrm{b}(v(x, \omega), u(x, \omega))) & =\mathbf{E}(\mathrm{b}(v(x), u(x, \omega))) \\
=\mathrm{b}(v(x), \mathbf{E}(u(x, \omega)))=\mathrm{b}(v, \bar{u})=\langle\langle f, v\rangle\rangle=\mathbf{E}(\langle f, v\rangle) & =\langle\mathbf{E}(f), v\rangle=\langle\bar{f}, v\rangle,
\end{aligned}
$$

and hence the mean $\bar{u}$ satisfies the deterministic Eq.(1) or variational problem Eq.(2), with the deterministic RHS or source $\bar{f}$. 
Similarly, for the covariance function $C_{u}(x, y)$ it may be seen by considering Eq.(14) at position $x$ and at position $y$, multiplying these two equations and taking the expected value, interchanging integration w.r.t. $\omega$ with differentiation w.r.t. $x$ and $y$ and using the linearity of the expectation, that it satisfies a differential equation on the domain $R \times R$, which has twice the number of spatial dimensions of the original problem Eq.(1). As $\stackrel{\circ}{H}{ }^{1}(R \times R) \cong \stackrel{\circ}{=} H^{1}(R) \otimes \stackrel{\circ}{H}{ }^{1}(R)=V \otimes V$, we may define the differential operator

$$
\nabla_{x} \otimes \nabla_{y}: V \otimes V \rightarrow Q \otimes Q \cong L_{2}\left(R \times R, \mathbb{R}^{d} \otimes \mathbb{R}^{d}\right)
$$

by only looking at products $v(x) w(y)$ with $v, w \in \stackrel{\circ}{H}^{1}(R)$, and then extending by linearity and continuity:

\section{Definition 2.}

$$
\nabla_{x} \otimes \nabla_{y}: v(x) w(y) \mapsto\left(\nabla_{x} v(x)\right) \otimes\left(\nabla_{y} w(y)\right)
$$

With this one may show the following proposition on the variational formulation $[3,14,65]$ :

Proposition 4. The covariance function $C_{u}(x, y)$ of the solution $u(x, \omega)$ to the linear stochastic RHS problem is the solution to the variational problem: Find $C_{u}$ such that for all $C_{v}(x, y) \in \stackrel{\circ}{H^{1}}(R \times R)$

$$
\begin{aligned}
& \iint_{R \times R}\left(\nabla_{x} \otimes \nabla_{y} C_{v}(x, y)\right): \kappa(x) \kappa(y)\left(\nabla_{x} \otimes \nabla_{y} C_{u}(x, y)\right) d x d y \\
&=\iint_{R \times R} C_{v}(x, y) C_{f}(x, y) d x d y
\end{aligned}
$$

where $C_{f}(x, y)$ is the covariance function of the stochastic RHS $f(x, \omega)$,

and where the ":"-operation is a contraction over both indices.

\subsection{Discretisation and Numerical Approximation}

We assume that the spatial part of the SPDE has been approximated by a Galerkin method. In some sense an arbitrary spatial discretisation could be used, but as we deal with Galerkin methods in the stochastic domain, assuming this also in the spatial domain gives a certain measure of unity to the presentation. What we use in the discretisation amounts to the finite element method in space-it does not matter which variant - and a spectral or pure p-method in the stochastic dimension [22]. 
The consistency of such approximations-density of the union of finite dimensional subspaces-was shown in [8,70,14], and also convergence, although partly in another context, see also [4, 6, 7]. There one may find also à priori error estimates. We here use Bubnov-Galerkin methods on a symmetric and positive definite problem, hence stability is inherited from the coercivity of the continuous problem, and convergence is guaranteed by consistency alone (see e.g. [68]).

\subsubsection{Spatial Discretisation}

Performing a Galerkin approximation in the spatial part amounts to taking only a finite-dimensional subspace $V_{N} \subset V$. Let $\left\{s_{1}(x), \ldots, s_{N}(x)\right\}$ be a basis in $V_{N}$, we then approximate the solution by $[12,68,77]$

$$
u(x, \omega)=\sum_{k=1}^{N} s_{k}(x) u_{k}(\omega)=\boldsymbol{s}(x) \boldsymbol{u}(\omega),
$$

where the $\left\{u_{k}(\omega)\right\}$ now are random variables in $(S)$, and for conciseness of notation we have set $\boldsymbol{s}(x)=\left[s_{1}(x), \ldots, s_{N}(x)\right]$ and $\boldsymbol{u}(\omega)=\left[u_{1}(\omega), \ldots, u_{N}(\omega)\right]^{T}$.

Calculation of the mean $\bar{u}(x)$ is a purely deterministic and standard problem, and we shall not consider it here any further. We are concerned with the fluctuating part $\tilde{u}(x, \omega)$. This means that we approximate $\tilde{u}(x, \omega)$ with the ansatz as in Eq.(22), where we may take the coefficient random variables $u_{k}(\omega)$ to have zero mean.

Inserting this ansatz into Eq.(17), and applying the Galerkin conditions, where we take as test functions products like $\varphi(\omega) s_{\imath}(x)$ with $\varphi \in(S)$, we require that for all $\varphi \in(S)$ :

$$
\int_{\Omega} \varphi(\omega) \mathbf{K} \tilde{\boldsymbol{u}}(\omega) \Gamma(d \omega)=\mathbf{K} \int_{\Omega} \varphi(\omega) \tilde{\boldsymbol{u}}(\omega) \Gamma(d \omega)=\int_{\Omega} \varphi(\omega) \tilde{\boldsymbol{f}}(\omega) \Gamma(d \omega),
$$

where the spatial discretisation has already been completed.

$$
\mathbf{K}=\left(K_{\imath \jmath}\right)=\left(\int_{R}\left(\nabla s_{\imath}(x)\right)^{T} \kappa(x)\left(\nabla s_{\jmath}(x)\right) d x\right)
$$

is the usual deterministic stiffness matrix, and the RHS is

$$
\tilde{\boldsymbol{f}}(\omega)=\left[\tilde{f}_{1}(\omega), \ldots, \tilde{f}_{N}(\omega)\right]^{T}
$$

with $\tilde{f}_{\jmath}(\omega)=\int_{R} s_{\jmath}(x) \tilde{f}(x, \omega) d x$. The variational Eq.(23) will be written as

$$
\mathbf{K} \tilde{\boldsymbol{u}}(\omega)=\tilde{\boldsymbol{f}}(\omega)
$$


understood in a weak sense, meaning that for all $\varphi(\omega) \in(S)$ :

$$
\mathbf{E}(\varphi(\omega) \mathbf{K} \tilde{\boldsymbol{u}}(\omega))=\mathbf{K} \mathbf{E}(\varphi(\omega) \tilde{\boldsymbol{u}}(\omega))=\mathbf{E}(\varphi(\omega) \tilde{\boldsymbol{f}}(\omega)),
$$

whereas the mean $\overline{\boldsymbol{u}}$ is a solution of

$$
\mathbf{K} \bar{u}=\bar{f} .
$$

It is now left to solve the stochastic linear system of Eq.(24). This still involves the variable $\omega \in \Omega$, and is still computationally intractable, as in general we need infinitely many coordinates to parametrise $\Omega$.

\subsubsection{Stochastic Discretisation}

In section 2.2.2 we used the PC-expansion to represent a stochastic process. Here we want to extend the Galerkin idea, and for that reason we expand the random variables $\tilde{\boldsymbol{u}}(\omega)^{T}=\left[\tilde{u}_{1}(\omega), \ldots, \tilde{u}_{N}(\omega)\right]$ in a PC-series:

$$
\forall k: \quad \tilde{u}_{k}(\omega)=\sum_{\alpha} u_{k}^{\alpha} H_{\alpha}(\omega)=\mathrm{u}_{k} \mathrm{H}(\omega),
$$

where $\mathrm{H}(\omega)^{T}=\left[\ldots, H_{\alpha}(\omega), \ldots\right]$, and $\mathrm{u}_{k}=\left[\ldots, u_{k}^{\alpha}, \ldots\right]$.

For the purpose of actual computation, we will truncate the PC-expansion Eqs.(10,27) after finitely many terms $\alpha \in \mathcal{J}_{M, p}$, thus introducing a finite dimensional approximation $\operatorname{span}\left\{H_{\alpha} \mid \alpha \in \mathcal{J}_{M, p}\right\} \subset(S)$. The set $\mathcal{J}_{M, p} \subset \mathcal{J}$ is here defined for $M, p \in \mathbb{N}$ as (see also $[22,8,70]$ ):

$$
\mathcal{J}_{M, p}=\left\{\alpha \in \mathcal{J}\left|\forall \jmath>M: \alpha_{\jmath}=0,\right| \alpha \mid \leq p\right\} .
$$

Just as we require for $\bigcup_{N} V_{N}$ to be dense in $\stackrel{\circ}{H}^{1}(R)$ — see $[68,12]$ - here we rely on the fact that the closure of $\bigcup_{M, p} \operatorname{span}\left\{H_{\alpha} \mid \alpha \in \mathcal{J}_{M, p}\right\}$ is all of $(S)$, see section 2.2.2

\subsection{Computational Procedures}

Even after the discretisation has been spelled out in the preceeding sections 3.2.1 and 3.2.2, there still remain many choices on how to actually organise the computation. Some of them may be seen as discretisations in their own right, or when applied to an already finite dimensional problem they could be more appropriately labelled as methods to reduce the dimensionality of the system. As usually stochastic problems are addressed with the Monte Carlo method, we give 
here a quick summary, and we use some of these ideas later in a different setting. Subsequently, we show three different ways of making the approximation of the stochastic part; these are the pure polynomial chaos (PC) expansion in section 3.3.2, with the Karhunen-Loève expansion of the RHS in section 3.3.3, and finally with the Karhunen-Loève expansion of the solution itself in section 3.3.4.

\subsubsection{Monte Carlo Methods}

Let us touch shortly on the Monte Carlo (MC) method, as it would be often the natural first choice for anyone faced with solving a stochastic PDE. Both MC and its relative, the Quasi Monte Carlo (QMC) method are based on the following idea: If the aim is to compute a functional of the solution such as in Eq.(15), one would try to approximate the integral numerically:

$$
\Psi_{u}(x) \approx \sum_{z=1}^{Z} w_{z} \Psi\left(\boldsymbol{s}(x)\left(\boldsymbol{u}\left(\omega_{z}\right)\right),\right.
$$

where $\boldsymbol{s}(x)$ are the spatial FEM-basis, $\omega_{z}$ are the evaluation points, and $w_{z}$ are the weights-in MC- and QMC-methods the weights are $w_{z}=1 / Z$. We would like to stress once more that all the statistics which are usually considered are really such integrals of a function of $\boldsymbol{u}(\omega)$ over $\Omega$.

The problem is that we do not know $\boldsymbol{u}(\omega)$, as this is the still unknown solution. But Eq.(29) does not really require that we know $\boldsymbol{u}(\omega)$ for all $\omega$, i.e. that we have a functional expression for it. All we have to know is the solution at the particular evaluation points $\omega_{z}$, which means the solution for particular realisations of the random field, in this case $f\left(x, \omega_{z}\right)$. Once we have fixed $\omega=\omega_{z}$, the problem is completely deterministic, and may be solved by standard methods.

To compute this integral, we may proceed in the following way:

1. Select the points $\left\{\omega_{z} \mid z=1, \ldots, Z\right\} \subset \Omega$ according to the integration rule.

2. For each $\omega_{z}$-a realisation of the stochastic system—solve the deterministic problem with that fixed realisation, yielding $\boldsymbol{u}\left(\omega_{z}\right)$.

3. For each $\omega_{z}$ compute the integrand $\Psi_{u}\left(\boldsymbol{s}(x) \boldsymbol{u}\left(\omega_{z}\right)\right)$. in Eq.(29).

4. Perform the summation in Eq.(29).

Note the potential for "embarrassingly easy" parallelisation in steps 2 and 3.

In this light, the Monte Carlo method proceeds in the following way: In step 2 of the above procedure, the selection is to take a number $Z$ of independent random samples $\left\{\omega_{z} \mid z=1, \ldots, Z\right\} \subset \Omega$ according to the measure $\Gamma$ - this makes sure that the points all carry the same weight $w_{z}=1 / Z$, see [10]. 
The Quasi Monte Carlo method is only slightly different from this point of view, as all the difference is that the points $\left\{\omega_{z}\right\}$ are selected from a "low discrepancy set", still all with weights $w_{z}=1 / Z$, see $[51,10]$.

Note that this form of computation is completely independent of what kind of stochastic problem we have, be it linear or nonlinear, or with stochastic RHS or stochastic operator. This is on one hand the strength of this approach-its general applicability, but on the other hand it does not take into account any special properties of the problem. The methods to be described in the next sections will take such special properties into account, and can therefore be much more efficient.

\subsubsection{Using the Polynomial Chaos Expansion}

A PC-expansion similar to Eq.(10) allows us to write Eqs. $(23,24,25)$ with an expansion of both $\tilde{\boldsymbol{u}}$ and $\tilde{\boldsymbol{f}}$ (see [22]):

$$
\begin{aligned}
\mathbf{K} \tilde{\boldsymbol{u}}(\omega) & =\mathbf{K}\left(\sum_{\alpha \in \mathcal{J}} \boldsymbol{u}^{(\alpha)} H_{\alpha}(\omega)\right)=\sum_{\alpha \in \mathcal{J}} \mathbf{K} \boldsymbol{u}^{(\alpha)} H_{\alpha}(\omega) \\
& =\tilde{\boldsymbol{f}}(\omega)=\sum_{\alpha \in \mathcal{J}} \boldsymbol{f}^{(\alpha)} H_{\alpha}(\omega) .
\end{aligned}
$$

As the representation is unique, we have proven the next proposition.

Proposition 5. The coefficients $\boldsymbol{u}^{(\alpha)}$ in the PC-expansion of

$$
\tilde{\boldsymbol{u}}(\omega)=\sum_{\alpha \in \mathcal{J}} \boldsymbol{u}^{(\alpha)} H_{\alpha}(\omega)
$$

satisfy the uncoupled system of equations

$$
\forall \alpha \in \mathcal{J}: \mathbf{K} \boldsymbol{u}^{(\alpha)}=\boldsymbol{f}^{(\alpha)} .
$$

This means that the coefficients in the polynomial chaos expansion may be computed one-by-one and independently of each other-and hence in parallel with no interaction. This is due to the orthonormality of the polynomial chaos basis. As the set $\mathcal{J}$ is infinite, in an actual computation we take a finite subset such as $\mathcal{J}_{M, p} \subset \mathcal{J}$ defined in relation Eq.(28), and compute only those $\boldsymbol{u}^{(\alpha)}$ with $\alpha \in \mathcal{J}_{M, p}$.

Remark 1. Once we have the $\boldsymbol{u}^{(\alpha)}$, we may compute approximately any statistic Eq.(15) of u, and especially $-\bar{u}$ being already approximated by $\overline{\boldsymbol{u}}$-the covariance

$$
C_{u}(x, y) \approx \boldsymbol{s}(x) \mathbf{E}(\tilde{\boldsymbol{u}}(\omega) \otimes \tilde{\boldsymbol{u}}(\omega)) \boldsymbol{s}^{T}(y)=\boldsymbol{s}(x) \mathbf{C}_{u} \boldsymbol{s}^{T}(y),
$$


where

$$
\mathbf{C}_{u}:=\mathbf{E}(\tilde{\boldsymbol{u}}(\omega) \otimes \tilde{\boldsymbol{u}}(\omega))=\sum_{\alpha \in \mathcal{J}_{M, p}} \boldsymbol{u}^{(\alpha)} \otimes \boldsymbol{u}^{(\alpha)}
$$

is the discrete covariance matrix of $\tilde{\boldsymbol{u}}(\omega)$.

Remark 2. We could have used the PC-expansion already in the continuous case

$$
u(x, \omega)=\sum_{\alpha} u^{(\alpha)}(x) H_{\alpha}(\omega), \quad f(x, \omega)=\sum_{\alpha} f^{(\alpha)}(x) H_{\alpha}(\omega)
$$

—where the coefficients are given by $f^{(\alpha)}(x):=\mathbf{E}\left(f(x, \cdot) H_{\alpha}(\cdot)\right)$ —and we would have seen that each $u^{(\alpha)}(x)$ satisfies the same PDE Eqs. $(1,2)$, but with (deterministic) $R H S f^{(\alpha)}(x)$. This is the continuous analogue of Eq.(32).

Remark 3. The $f^{(\alpha)}(x)$ may be smoother than $f(x, \omega)$ and hence a coarser spatial discretisation could suffice, or it could be different for each $\alpha$. For the sake of simplicity we shall not explore this possibility further and assume that the spatial discretisation is always the same.

Certainly the $f^{(\alpha)}(x)$ or $\boldsymbol{f}^{(\alpha)}$ have to be given or computed somehow, and a potential problem is that we may not have an explicit expression for $f(x, \omega)$ with which to compute it. Also, in the discrete case, there can be only $N$ linearly independent $\boldsymbol{f}^{(\alpha)}-N=\operatorname{dim} V_{N}$ is the number of spatial basis functions-so one ought to do better. One possibility is the Karhunen-Loève (KL) expansion.

\subsubsection{Using the Karhunen-Loève Expansion}

The Karhunen-Loève (KL) expansion alluded to in the previous section 3.3.2 opens up another possibility. Writing the already spatially discretised KLexpansion of $\tilde{\boldsymbol{f}}(\omega)$ as

$$
\tilde{\boldsymbol{f}}(\omega)=\sum_{\ell} \varphi_{\ell}(\omega) \boldsymbol{f}_{\ell}
$$

we insert this into Eq.(25), and we obtain

$$
\tilde{\boldsymbol{u}}(\omega)=\mathbf{K}^{-1} \tilde{\boldsymbol{f}}(\omega)=\mathbf{K}^{-1}\left(\sum_{\ell} \varphi_{\ell}(\omega) \boldsymbol{f}_{\ell}\right)=\sum_{\ell} \varphi_{\ell}(\omega) \mathbf{K}^{-1} \boldsymbol{f}_{\ell} .
$$

This implies the following result: 
Proposition 6. Each $\boldsymbol{u}^{(\alpha)}$ in Eq.(30) may be computed as a linear combination of the vectors $\hat{\boldsymbol{u}}_{\ell}$, satisfying $\mathbf{K} \hat{\boldsymbol{u}}_{\ell}=\boldsymbol{f}_{\ell}$ :

$$
\begin{aligned}
\boldsymbol{u}^{(\alpha)} & =\mathbf{E}\left(H_{\alpha}(\cdot) \tilde{\boldsymbol{u}}(\cdot)\right)=\mathbf{E}\left(H_{\alpha}(\cdot) \sum_{\ell} \varphi_{\ell}(\cdot) \mathbf{K}^{-1} \boldsymbol{f}_{\ell}\right) \\
& =\sum_{\ell} \mathbf{E}\left(H_{\alpha}(\cdot) \varphi_{\ell}(\cdot)\right) \mathbf{K}^{-1} \boldsymbol{f}_{\ell}=\sum_{\ell} \varphi_{\ell}^{(\alpha)} \mathbf{K}^{-1} \boldsymbol{f}_{\ell}=\sum_{\ell} \varphi_{\ell}^{(\alpha)} \hat{\boldsymbol{u}}_{\ell} .
\end{aligned}
$$

Remark 4. A remark similar to Remark 3 applies here as well: The continuous analogues of $\boldsymbol{f}_{\ell}$, the KL-eigenfunctions of $C_{f}(x, y)$, are often much smoother than $f$ itself; hence the spatial discretisation may be reduced, or even better adapted individually for each $\boldsymbol{f}_{\ell}$. Again, for the sake of simplicity of exposition, we shall assume that the spatial discretisation is always the same.

That means that we can solve-see [18] —independently and in parallel without communication-for the vectors $\hat{\boldsymbol{u}}_{\ell}$, and they represent $\tilde{\boldsymbol{u}}(\omega)$ as

$$
\tilde{\boldsymbol{u}}(\omega)=\sum_{\alpha} \boldsymbol{u}^{(\alpha)} H_{\alpha}(\omega)=\sum_{\alpha} \sum_{\ell} \varphi_{\ell}^{(\alpha)} H_{\alpha}(\omega) \hat{\boldsymbol{u}}_{\ell}
$$

Remark 5. Looking at Eq.(11), it is obvious that for each KL-term there will be at least one PC-term, so there are less solves in Eq.(34) for the KL-expansion than in Eq.(31) for the pure PC-expansion.

If we use all $N$ eigenvectors, there is no difference to the solution in the previous subsection, other than that the computations are arranged differently. But the idea is to have many fewer vectors. The problem is that the choice based on the spectrum of $C_{f}(x, y)$ alone does not tell us how important they are in the solution $\boldsymbol{u}(\omega)$, as we have not taken account of the transfer properties of the system. Best would be of course to use the KL-expansion of the solution $\boldsymbol{u}(\omega)$. We address this in the next section.

\subsubsection{Using the Karhunen-Loève Expansion of the Solution}

The problem is of course here that we do not know the KL-expansion of $\boldsymbol{u}(\omega)$, as we do not know $\boldsymbol{u}(\omega)$ itself, we do not even know the covariance $\mathbf{C}_{u}=$ $\mathbf{E}(\tilde{\boldsymbol{u}}(\cdot) \otimes \tilde{\boldsymbol{u}}(\cdot))$ of $\boldsymbol{u}(\omega)$, wherefrom we might compute the KL-basis. Certainly, from Eq.(21) in Proposition 4, we see that [65]:

Proposition 7. The covariance matrix $\mathbf{C}_{u}$ is the solution of

$$
(\mathbf{K} \otimes \mathbf{K}) \mathbf{C}_{u}=\mathbf{K C}_{u} \mathbf{K}^{*}=\mathbf{C}_{f}
$$


This corresponds to solving the PDE Eq.(21) on the domain $R \times R \subset \mathbb{R}^{2 d}$, which is a large effort even when sparse grid methods are used [65].

We want to propose a simpler and more effective approach, and address this problem by first solving an auxiliary generalised eigenproblem which involves directly both $\mathbf{K}$ and $\mathbf{C}_{f}$, namely

$$
\mathbf{K} \boldsymbol{w}_{m}=\theta_{m} \mathbf{C}_{f} \boldsymbol{w}_{m}
$$

or in Matrix form

$$
\mathbf{K W}=\mathbf{C}_{f} \mathbf{W} \Theta
$$

where $\mathbf{W}=\left[\boldsymbol{w}_{1}, \ldots, \boldsymbol{w}_{m}, \ldots, \boldsymbol{w}_{M}\right]$ and $\boldsymbol{\Theta}=\operatorname{diag}\left(\theta_{m}\right)$. As both $\mathbf{K}$ and $\mathbf{C}_{f}$ are symmetric and positive definite, we know that all the $\theta_{m}>0$, and

$$
\mathbf{W}^{*} \mathbf{K W}=\boldsymbol{\Theta}, \quad \mathbf{W}^{*} \mathbf{C}_{f} \mathbf{W}=\mathbf{I}_{M} .
$$

In contrast to solving Eq.(36), where all of $\mathbf{C}_{u}$ has to be computed, we can stop the eigenvalue extraction at $M \ll N$, and hence work with a low rank approximation of $\mathbf{C}_{u}$, as will be shown in Theorems 1 and 2 .

If we would take $M=N$, the last relation would imply that $\mathbf{W}$ is non-singular and we would have

$$
\mathbf{K}^{-1}=\mathbf{W} \Theta^{-1} \mathbf{W}^{*}, \quad \mathbf{C}_{f}=\mathbf{W}^{-*} \mathbf{W}^{-1},
$$

where $\mathbf{W}^{-*}=\left(\mathbf{W}^{-1}\right)^{*}$.

Theorem 1. For $M=N$, when $\mathbf{W}$ is non-singular, we have

$$
\mathbf{C}_{u}=\mathbf{W} \Theta^{-2} \mathbf{W}^{*}
$$

and for $M<N$ this is a rank $M$ approximation to the discrete covariance matrix.

Proof. We see from Eq.(36) that

$$
\mathbf{C}_{u}=(\mathbf{K} \otimes \mathbf{K})^{-1} \mathbf{C}_{f}=\mathbf{K}^{-1} \mathbf{W}^{-*} \mathbf{W}^{-1} \mathbf{K}^{-*},
$$

and from Eq.(40) we substitute $\mathbf{K}^{-1}$ to obtain

$$
\mathbf{C}_{u}=\mathbf{W} \Theta^{-1} \mathbf{W}^{*} \mathbf{W}^{-*} \mathbf{W}^{-1} \mathbf{W} \Theta^{-1} \mathbf{W}^{*}=\mathbf{W} \Theta^{-2} \mathbf{W}^{*} .
$$


We take this as a guide and set also in the case $M<N$ as low rank approximation $\hat{\mathbf{C}}_{u}=\mathbf{W \Theta}^{-2} \mathbf{W}^{*}$. A little calculation shows that

$$
\mathbf{W}^{*} \mathbf{K} \hat{\mathbf{C}}_{u} \mathbf{K}^{*} \mathbf{W}=\mathbf{W}^{*} \mathbf{C}_{f} \mathbf{W}=\mathbf{I}_{M},
$$

which may be seen as the defining relation.

In the computation we decide depending on the size of $\left|\theta_{m}\right|^{-2}$ where to stop, i.e. we compute the eigenvalues/vectors in Eq.(37) starting from the smallest, and stop when $\left|\theta_{m}\right|^{-2}$ is small enough at $m=M$. This is not yet the KL-decomposition of $\tilde{\boldsymbol{u}}$, as $\mathbf{W}$ is not orthogonal (not composed of orthogonal columns).

Computing the QR-decomposition of $\mathbf{W}=\mathrm{QR}$, we obtain

$$
\mathbf{C}_{u}=\mathbf{Q R} \Theta^{-2} \mathbf{R}^{*} \mathbf{Q}^{*}=\mathbf{Q}\left(\mathbf{R} \Theta^{-1}\right)\left(\Theta^{-1} \mathbf{R}^{*}\right) \mathbf{Q}^{*}=\mathbf{Q} \hat{\mathbf{R}} \hat{\mathbf{R}}^{*} \mathbf{Q}^{*}
$$

These considerations imply the following theorem:

Theorem 2. Solving the standard eigenproblem of size $M \times M$

$$
\mathbf{X} \hat{\mathbf{Q}}:=\hat{\mathbf{R}} \hat{\mathbf{R}}^{*} \hat{\mathbf{Q}}=\hat{\mathbf{Q}} \boldsymbol{\Lambda}_{u},
$$

we obtain the $K L$ - or eigendecomposition of

$$
\mathrm{C}_{u}=\mathrm{Q}_{\hat{\mathbf{R}}} \hat{\mathbf{R}}^{*} \mathrm{Q}^{*}=\mathrm{QXQ}^{*}=\mathrm{Q} \hat{\mathbf{Q}} \Lambda_{u} \hat{\mathrm{Q}}^{*} \mathrm{Q}^{*}=\boldsymbol{\Phi}_{u} \Lambda_{u} \boldsymbol{\Phi}_{u}^{*}
$$

with the spectrum on the diagonal of $\Lambda_{u}$ and the orthogonal eigenvector matrix $\boldsymbol{\Phi}_{u}=\mathbf{Q} \hat{\mathbf{Q}}=:\left[\boldsymbol{u}_{1}, \ldots, \boldsymbol{u}_{M}\right]$.

In the computation it may be used that we have the Cholesky decomposition of $\mathbf{X}=\hat{\mathbf{R}} \hat{\mathbf{R}}^{*}$, details of this will be published elsewhere.

We want to use these results now to compute the PC-coefficients of the solution. From Eq.(32) and the spectral decomposition of $\mathbf{C}_{f}=\boldsymbol{\Phi}_{f} \boldsymbol{\Lambda}_{f} \boldsymbol{\Phi}_{f}^{*}$, we see that

$$
\boldsymbol{u}^{(\alpha)}=\mathbf{K}^{-1} \boldsymbol{f}^{(\alpha)}=\mathbf{K}^{-1} \boldsymbol{\Phi}_{f} \boldsymbol{\varphi}^{(\alpha)},
$$

where $\boldsymbol{\varphi}^{(\alpha)}=\left[\ldots, \phi_{\ell}^{(\alpha)}, \ldots\right]^{T}$. But $\mathbf{K}^{-1} \boldsymbol{\Phi}_{f}$ does not have to computed, as from $\mathbf{W}=\mathbf{K}^{-1} \mathbf{C}_{f} \mathbf{W} \boldsymbol{\Theta}=\left(\mathbf{K}^{-1} \boldsymbol{\Phi}_{f}\right) \boldsymbol{\Lambda}_{f} \boldsymbol{\Phi}_{f}^{*} \mathbf{W} \boldsymbol{\Theta}$ we see that $\mathbf{K}^{-1} \boldsymbol{\Phi}_{f}$ is in the column span of $\mathbf{W}$.

Setting $\boldsymbol{u}^{(\alpha)}=\mathbf{W} \hat{\boldsymbol{u}}^{(\alpha)}$, we multiply by $\mathbf{W}^{*} \mathbf{K}$ and obtain

$$
\mathbf{W}^{*} \mathbf{K} \mathbf{W} \hat{\boldsymbol{u}}^{(\alpha)}=\boldsymbol{\Theta} \hat{\boldsymbol{u}}^{(\alpha)}=\mathbf{W}^{*} \boldsymbol{\Phi}_{f} \boldsymbol{\varphi}^{(\alpha)} .
$$

We collect these results in the following proposition: 
Proposition 8. The PC-vectors $\boldsymbol{u}^{(\alpha)}$ may be approximated by

$$
\boldsymbol{u}^{(\alpha)}=\mathbf{W} \Theta^{-1} \mathbf{W}^{*} \Phi_{f} \varphi^{(\alpha)} .
$$

Here $\mathbf{W} \Theta^{-1} \mathbf{W}^{*}$ is rank $M$ approximation to $\mathbf{K}^{-1}$. In this approach we have obtained a low rank approximation to $\mathbf{C}_{u}$ as a by-product-by solving a generalised eigenproblem - and we see that at least enough eigen- or KL-vectors of $\mathbf{C}_{f}$ have to be used, such that $\mathbf{W}^{*} \boldsymbol{\Phi}_{f}$ has full rank.

In either of the three solution approaches considered it seems easier to first compute $\boldsymbol{u}^{(\alpha)}$, and from that $\mathbf{C}_{u}$, than to solve for $\mathbf{C}_{u}$ directly-in the third approach $\mathrm{C}_{u}$ is part of the solution anyway, see Eq.(41).

If we only want the covariance

$$
\mathbf{C}_{u}=(\mathbf{K} \otimes \mathbf{K})^{-1} \mathbf{C}_{f}=\left(\mathbf{K}^{-1} \otimes \mathbf{K}^{-1}\right) \mathbf{C}_{f}=\mathbf{K}^{-1} \mathbf{C}_{f} \mathbf{K}^{-*}
$$

one may-with the KL-expansion of $\mathbf{C}_{f}=\boldsymbol{\Phi}_{f} \Lambda_{f} \boldsymbol{\Phi}_{f}^{*}$ and by solving first $\hat{\boldsymbol{\Phi}}=$ $\mathbf{K}^{-1} \boldsymbol{\Phi}_{f}$, where the columns of $\hat{\boldsymbol{\Phi}}=\left[\ldots, \hat{\boldsymbol{u}}_{\ell}, \ldots\right]$ are the vectors from Eq.(35) -certainly reduce this to

$$
\mathbf{C}_{u}=\mathbf{K}^{-1} \mathbf{C}_{f} \mathbf{K}^{-*}=\mathbf{K}^{-1} \boldsymbol{\Phi}_{f} \boldsymbol{\Lambda}_{f} \boldsymbol{\Phi}_{f}^{*} \mathbf{K}^{-*}=\hat{\boldsymbol{\Phi}} \Lambda_{f} \hat{\boldsymbol{\Phi}}
$$

Any of these approaches seems easier than directly discretising the PDE in Eq.(21) in $\mathbb{R}^{2 d}$ according to Proposition 7 (cf. [65]) —the discrete analogue of this is Eq.(48).

\subsubsection{Direct or Non-Intrusive Computation}

Note that the expansion coefficients $\boldsymbol{u}^{(\alpha)}$ can also be seen as a functional or statistic of the solution. We have

Proposition 9. The coefficients $\boldsymbol{u}^{(\alpha)}$ are given by

$$
\begin{aligned}
\boldsymbol{u}^{(\alpha)}=\mathbf{E}\left(\tilde{\boldsymbol{u}}(\cdot) H_{\alpha}(\cdot)\right)=\mathbf{E}\left(\mathbf{K}^{-1} \tilde{\boldsymbol{f}}(\cdot) H_{\alpha}(\cdot)\right) & \\
& =\int_{\Omega} \mathbf{K}^{-1} \tilde{\boldsymbol{f}}(\omega) H_{\alpha}(\omega) \Gamma(d \omega) .
\end{aligned}
$$

Certainly this can be computed by the methods described in section 3.3.1. We borrow the term non-intrusive from [23] for this way of evaluating the PCexpansion. Please note that this way of evaluating the coefficients is completely independent of the type of stochastic system, similar to the comment already made in conjunction with the Monte Carlo method in section 3.3.1. 
We approximate this by allowing only $\alpha \in \mathcal{J}_{M, p}$. Then Eq.(50) reduces to an integral over the finite dimensional space $\Omega_{m}=\operatorname{span}\left\{\omega_{j}\right\}$, and the mutual independence allows the application of Fubini's theorem and the integral is reduced to $m$ iterated one-dimensional integrals. This integral may be computed via any integration method including Monte Carlo or Quasi Monte Carlo methods. See [36] for a study when it is advisable to use what kind of integration.

In Eq.(50) it is clear that

$$
\int_{\Omega} \mathbf{K}^{-1} \tilde{\boldsymbol{f}}(\omega) H_{\alpha}(\omega) \Gamma(d \omega)=\mathbf{K}^{-1}\left(\int_{\Omega} \tilde{\boldsymbol{f}}(\omega) H_{\alpha}(\omega) \Gamma(d \omega)\right)=\mathbf{K}^{-1} \boldsymbol{f}^{(\alpha)},
$$

and there is really no point in trying to use a direct or non-intrusive method here for our special case of only a stochastic RHS.

\subsection{The Solution as Filtered White Noise}

We can go one step further in describing the effect of the linear system to the stochastic input. We would like to see it as a filter for white noise. From any of the possible paths described in the previous section we obtain the coefficients $\boldsymbol{u}^{(\alpha)}$ of the PC-expansion

$$
\boldsymbol{u}(\omega)=\overline{\boldsymbol{u}}+\sum_{\alpha} \boldsymbol{u}^{(\alpha)} H_{\alpha}(\omega)=\overline{\boldsymbol{u}}+\mathbf{u H}(\omega),
$$

with $\mathbf{u}=\left[\ldots, \boldsymbol{u}^{(\alpha)}, \ldots\right]$. Now $\boldsymbol{\omega}=\left(\omega_{1}, \ldots, \omega_{M}\right)$ is our white noise process, and the above representation shows how $\boldsymbol{u}(\omega)$ is composed from a linear combination of (Hermite)-polynomial filters. Certainly, if the input $\boldsymbol{f}(\omega)$ is Gaussian, the output - a linear deterministic function of the input-is also Gaussian. This means that in Eq.(51) the sum is only over $|\alpha| \leq 1$, and so is reduced to

$$
\boldsymbol{u}(\omega)=\overline{\boldsymbol{u}}+\sum_{\jmath} \boldsymbol{u}^{(\jmath)} \omega_{\jmath}=\overline{\boldsymbol{u}}+\mathbf{u} \boldsymbol{\omega},
$$

with $\mathbf{u}=\left[\boldsymbol{u}^{(1)}, \ldots, \boldsymbol{u}^{(M)}\right]$.

The representation in terms of the KL-expansion of $\boldsymbol{u}$, Eq.(47), shows now the way the linear system acts, as $\mathbf{u}=\mathbf{W} \Theta^{-1} \mathbf{W}^{*} \boldsymbol{\Phi}_{f} \mathrm{~F}$ (with $\mathrm{F}=\left[\boldsymbol{f}^{(1)}, \ldots, \boldsymbol{f}^{(M)}\right]$ ) and where $\mathrm{F}$ may be decomposed even further to $\mathrm{F}=\Lambda_{f}^{1 / 2} \tilde{\mathrm{F}}$, with, $\tilde{\mathrm{F}} \tilde{\mathrm{F}}^{*}=\mathbf{I}_{M}$. This results in

$$
\mathbf{u}=\mathbf{W} \boldsymbol{\Theta}^{-1} \mathbf{W}^{*} \boldsymbol{\Phi}_{f} \boldsymbol{\Lambda}_{f}^{1 / 2} \tilde{\mathrm{F}}=\mathbf{S} \tilde{\mathrm{F}}
$$

where $\mathbf{S}=\mathbf{W} \boldsymbol{\Theta}^{-1} \mathbf{W}^{*} \boldsymbol{\Phi}_{f} \Lambda_{f}^{1 / 2}$ is the transfer function to unit variance input.

We see the orthogonal mixing/superposition without change of variance-by $\tilde{\mathrm{F}}$, then the shaping by the spectrum of $\mathbf{C}_{f}$. Again orthogonal mixing due to $\boldsymbol{\Phi}_{f}$ - the KL-expansion - and then the action of the linear system by projecting onto the $\mathbf{W}$, shaping by $\Theta^{-1}$, and again using mixing/superposition with $\mathbf{W}$. 


\section{Linear Systems with Stochastic Operator -Multiplicative Noise}

We now go up one level higher in the difficulty level and consider a linear partial differential equation with a stochastic coefficient, satisfying the relation in Eq.(12):

$$
\begin{aligned}
-\nabla_{\omega}^{T} \cdot\left(\kappa(x, \omega) \nabla_{\omega} u(x, \omega)\right. & =f(x, \omega) \text { for } x \in R, \\
u(x) & =0 \text { for } x \in \partial R .
\end{aligned}
$$

We formulate this SPDE in a variational statement, one which involves also the stochastic variables $[71,8,70,14,4]$.

\subsection{Mathematical Formulation}

The weak form is to find a $u \in V \otimes(S)$, such that for all $v \in V \otimes(S)$ :

$$
\overline{\mathbf{b}}(v, u)=\mathbf{E}(\langle f, v\rangle)=:\langle\langle f, v\rangle\rangle,
$$

where

$$
\overline{\mathbf{b}}(v, u)=\mathbf{E}\left(\int_{R}\left(\nabla_{\omega} v(x, \omega)\right)^{T} \kappa(x, \omega)\left(\nabla_{\omega} u(x, \omega)\right) d x\right) .
$$

We have not completely specified what conditions $\kappa(x, \omega)$ has to satisfy, the choice of $(S)$ depends on this and on the RHS or source terms.

If $\kappa \in L_{\infty}(R) \otimes L_{\infty}(\Omega)$, we may choose $(S)=L_{2}(\Omega)$, for other choices see [9]. Remembering that we suppose the condition in Eq.(12) $\left(\kappa(x, \omega) \geq \kappa_{0}\right)$, we have [14]:

Proposition 10. For $f \in V^{*} \otimes(S)$ the problem Eq.(54) has a unique solution $u \in V \otimes(S)$, which depends continuously on $f$ and on $\kappa$.

Proof. Standard arguments show that $\mathbf{E}(\langle f, \cdot\rangle)$ is continuous on $V \otimes(S)$, and that $\overline{\mathbf{b}}(\cdot, \cdot)$ is continuous and coercive. The proposition is thus proven by application of the Lax-Milgram lemma. The continuous dependence on $\kappa$ in the $L_{\infty}$-norm may be seen as a consequence of the first Strang lemma (see [68, 12], and also $[4,6,7])$.

Corollary 3. Again this defines a continuous, self-adjoint, and positive definite operator $\overline{\mathbf{B}}: V \otimes(S) \rightarrow V^{*} \otimes(S)^{*}$ with continuous inverse. Both the operator and its inverse depend continuously on $\kappa$. In this view the stochastic variational problem Eq.(54) may be written as $\overline{\mathbf{B}} u=f$. 
Again we have satisfied all of Hadamard's requirements for a well-posed problem. It is still an open problem what conditions to impose in a stochastic sense on the random field $\kappa(x, \omega)$, in order for the conditions in the above variational setting to be satisfied.

\subsection{Discretisation and Numerical Approximation}

A standard spatial discretisation like in Eq.(23) leads to a problem of the form

$$
\mathbf{K}(\omega) \boldsymbol{u}(\omega)=\boldsymbol{f}(\omega)
$$

where both the matrix $\mathbf{K}(\omega)$ and the RHS $\boldsymbol{f}(\omega)$ are random variables, and hence so is $\boldsymbol{u}(\omega)$. We shall again attempt to write the solution $\boldsymbol{u}(\omega)$ as a linear combination of Hermite polynomials in $\omega$

$$
\boldsymbol{u}(\omega)=\sum_{\alpha} \boldsymbol{u}^{(\alpha)} H_{\alpha}(\omega),
$$

and the task will be again to compute the $\boldsymbol{u}^{(\alpha)}$. The Eq.(56) may be termed as one where we have both additive and multiplicative noise.

Often it is justified to assume that the random variables $\mathbf{K}(\omega)$ and $\boldsymbol{f}(\omega)$ are independent, hence $\Omega=\Omega_{1} \dot{\cup} \Omega_{2}$, and $\mathbf{K}(\omega)$ is a random variable only on $\Omega_{1}$, whereas $\boldsymbol{f}(\omega)$ is a random variable only on $\Omega_{2}$, so that

$$
\mathbf{K}\left(\omega_{1}\right) \boldsymbol{u}\left(\omega_{1}, \omega_{2}\right)=\boldsymbol{f}\left(\omega_{2}\right), \quad \omega_{1} \in \Omega_{1}, \omega_{2} \in \Omega_{2}
$$

The solution in that case may in a more refined way be written as

$$
\boldsymbol{u}\left(\omega_{1}, \omega_{2}\right)=\sum_{\alpha_{1}} \sum_{\alpha_{2}} \boldsymbol{u}^{\left(\alpha_{1}, \alpha_{2}\right)} H_{\alpha_{1}}\left(\omega_{1}\right) H_{\alpha_{2}}\left(\omega_{2}\right) .
$$

Projecting this onto $H_{\alpha_{1}}\left(\omega_{1}\right)$, we have

$$
\begin{aligned}
\boldsymbol{u}^{\left(\alpha_{1}, \cdot\right)}\left(\omega_{2}\right)=\sum_{\alpha_{2}} & \boldsymbol{u}^{\left(\alpha_{1}, \alpha_{2}\right)} H_{\alpha_{2}}\left(\omega_{2}\right)=\mathbf{E}_{1}\left(\boldsymbol{u}\left(\cdot, \omega_{2}\right) H_{\alpha_{1}}(\cdot)\right) \\
= & \mathbf{E}_{1}\left(\mathbf{K}(\cdot)^{-1} \boldsymbol{f}\left(\omega_{2}\right) H_{\alpha_{1}}(\cdot)\right)=\mathbf{E}_{1}\left(\mathbf{K}(\cdot)^{-1} H_{\alpha_{1}}(\cdot)\right) \boldsymbol{f}\left(\omega_{2}\right),
\end{aligned}
$$

where $\mathbf{E}_{1}(\cdot)$ is the expectation w.r.t the variables $\omega_{1} \in \Omega_{1}$.

Each of the $\boldsymbol{u}^{\left(\alpha_{1}, \cdot\right)}\left(\omega_{2}\right)$ satisfies an equation of the type where only the RHS is stochastic. In other words, the Eq.(60) is just of the same type as considered in the previous section on additive noise or stochastic RHS. This we now know how to handle, and we may concentrate on

$$
\begin{aligned}
& \boldsymbol{u}^{\left(\cdot, \alpha_{2}\right)}\left(\omega_{1}\right)=\sum_{\alpha_{1}} \boldsymbol{u}^{\left(\alpha_{1}, \alpha_{2}\right)} H_{\alpha_{1}}\left(\omega_{1}\right)=\mathbf{E}_{2}\left(\boldsymbol{u}\left(\omega_{1}, \cdot\right) H_{\alpha_{2}}(\cdot)\right) \\
& =\mathbf{E}_{2}\left(\mathbf{K}\left(\omega_{1}\right)^{-1} \boldsymbol{f}(\cdot) H_{\alpha_{2}}(\cdot)\right)=\mathbf{K}\left(\omega_{1}\right)^{-1} \mathbf{E}_{2}\left(\boldsymbol{f}(\cdot) H_{\alpha_{2}}(\cdot)\right)=\mathbf{K}\left(\omega_{1}\right)^{-1} \boldsymbol{f}^{\left(\alpha_{2}\right)},
\end{aligned}
$$


which for each $\alpha_{2}$ is a system with purely multiplicative noise, i.e. where the RHS is deterministic

$$
\mathbf{K}(\omega) \boldsymbol{u}(\omega)=\boldsymbol{f}
$$

Later we shall give some more comments on the combined case, but for now we see that under our assumption we may separate the problem into one of additive noise, where only the RHS is stochastic, and one of pure multiplicative noise, where only the operator is stochastic.

As already remarked in the preceeding section 3.2, the consistency of these methods was shown in $[8,70]$, for convergence results of similar problems see also $[14,4,6,7]$. There one may find also à priori error estimates. We again use Bubnov-Galerkin methods, and ascertain uniform coercitivity or positive definiteness, which is not automatic here, thus assuring stability. Together with consistency we obtain convergence (e.g. see $[68,12])$.

\subsection{Computational Procedures}

Similarly as in section 3.3, we again explore different computational approaches. As now also the operator is approximated, not just by projecting it onto a finite dimensional subspace, but by using the KL- and PC-expansions on the coefficient functions, we have to be concerned with stability of the numerical approximation. Starting with the Monte Carlo method in section 4.3.1, some care has already to be exercised in order to preserve stability of the numerical approximation process. Following the previous order for the stochastic RHS in section 3.3, we next look at the pure PC-expansion in section 4.3.2. Although not stable by itself, in connection with a Galerkin projection it turns out to be stable. Next we look at the KL-expansion of the coefficient function in the operator in section 4.3.3, and again in conjunction with the Galerkin projection a stable procedure can be found. Finding a better basis for the solution is the theme of section 4.3.4, and what could be better than the KL-expansion of the solution? The final subsection 4.3.5 in this part continues the description of the direct evaluation of the coefficients of the PC-expansion by integration.

\subsubsection{Monte Carlo Methods-Continued}

In principle we could proceed like before in section 3.3.1. We generate samples $\omega_{z}$, solve the equations etc., as described before. One aspect deserves further consideration though: The realisations of the conductivity all have to satisfy the relation Eq.(12), i.e. the conductivity has to be positive and bounded away from zero and bounded above a.e. If we generate the realisations through either the pure 
PC-expansion Eq.(9), or through the combined KL- and PC-expansion Eq.(11), to be numerically feasible it has to be truncated to a finite number of terms. But as both expansions only converge in $L_{2}(\Omega)$, there is no guarantee that for a fixed $\omega_{z}$ the relation Eq.(12) can be satisfied. After truncating the PC-expansion it will certainly be violated for some $\omega_{z}$, as we are now dealing with a polynomial in $\omega$, and polynomials are not bounded. Thus we face the prospect of potential numerical instability, or we might not even be able to solve the problem for a particular realisation, as it may be singular. Our computational procedure now does not satisfy Hadamard's requirements any more at the discrete level. This was no issue before in section 3.3.1, as there the operator was deterministic.

Even if in Eq.(5) the random variables $\xi_{k}(\omega)$ (see Eq.(6)) could be computed exactly and not through a truncated PC-expansion, there could still be problems, as also the KL-expansion only converges in $L_{2}(R)$, and has to be truncated in an actual computation. As already stated in section 2.2.1, with more requirements on the covariance function this convergence may be uniform (cf. also [6]).

Due to these arguments, we recommend in the case when particular realisations are computed to use the representation Eq.(3) with a pointwise transformation of a Gaussian field, in our examples of such direct simulations we used Eq.(13). The representation Eq.(3) makes sure that the relation Eq.(12) is satisfied in all cases. We shall see in the next section 4.3.2 that this difficulty may be avoided in conjunction with Galerkin projections.

\subsubsection{Can we use the Polynomial Chaos Expansion?}

In the case of additive noise we started first by looking at the polynomial chaos expansion of the stochastic noise term, in the spatially discretised version $\boldsymbol{f}(\omega)$. We may do the same here for the coefficient $\kappa(x, \omega)$, and in the discretised version for the matrix $\mathbf{K}(\omega)$ (cf. Eq.(9). As the stiffness matrix $\mathbf{K}(\omega)$ depends linearly on the conductivity $\kappa(x, \omega)$, we obtain a similar PC-expansion to Eq.(9) for the matrix:

$$
\mathbf{K}(\omega)=\sum_{\gamma} \mathbf{K}^{(\gamma)} H_{\gamma}(\omega)
$$

where of course $\mathbf{K}^{(0)}=\mathbf{E}(\mathbf{K}(\cdot))=\overline{\mathbf{K}}$, and each $\mathbf{K}^{(\gamma)}$ is a stiffness matrix computed with a "conductivity" $\kappa^{(\gamma)}(x)$ from Eq.(9).

Assuming also a PC-expansion of $\boldsymbol{u}(\omega)$ as in Eq.(27) in section 3.2.2 when we were dealing with a stochastic RHS alone, or as in Eq.(64) in the preceeding section 4.3.2, we have

$$
\left(\sum_{\gamma} \mathbf{K}^{(\gamma)} H_{\gamma}(\omega)\right) \sum_{\alpha} \boldsymbol{u}^{(\alpha)} H_{\alpha}(\omega)=\boldsymbol{f} .
$$


One potential worry here is-alluded to already in section 4.3.1 - that the PCexpansion in Eq.(9) converges only in $L_{2}(\Omega)$. We shall deal with this question as soon as we have performed the Galerkin projection.

To obtain a finite dimensional approximation, we truncate the series for $\boldsymbol{u}(\omega)$ by letting only $\alpha \in \mathcal{J}_{M, p}$ - this subset was defined in relation Eq.(28). If we do that, of course the equation can not be identically satisfied any more, and we use Galerkin projection conditions to obtain a unique solution.

$$
\begin{array}{r}
\forall \beta \in \mathcal{J}_{M, p}: \quad \mathbf{E}\left(H_{\beta}(\cdot)\left(\sum_{\gamma \in \mathcal{J}} \mathbf{K}^{(\gamma)} H_{\gamma}(\cdot)\right) \sum_{\alpha \in \mathcal{J}_{M, p}} \boldsymbol{u}^{(\alpha)} H_{\alpha}(\cdot)\right) \\
=\sum_{\alpha \in \mathcal{J}_{M, p}}\left(\sum_{\gamma \in \mathcal{J}} \mathbf{E}\left(H_{\beta}(\cdot) H_{\gamma}(\cdot) H_{\alpha}(\cdot)\right) \mathbf{K}^{(\gamma)}\right) \boldsymbol{u}^{(\alpha)} \\
=\mathbf{E}\left(H_{\beta}(\cdot) \boldsymbol{f}\right)=\boldsymbol{f}^{(\beta)} .
\end{array}
$$

Remark 6. Under the conditions assumed (deterministic RHS) we have $\boldsymbol{f}^{(\beta)}=$ $\delta_{0 \beta} \boldsymbol{f}^{(\beta)}$, i.e. only $\boldsymbol{f}^{(0)} \neq 0$, while all other $\boldsymbol{f}^{(\beta)}=0$.

Defining new matrices $\Delta^{(\gamma)}$ with elements

$$
\left(\boldsymbol{\Delta}^{(\gamma)}\right)_{\alpha, \beta}=\mathbf{E}\left(H_{\beta}(\cdot) H_{\gamma}(\cdot) H_{\alpha}(\cdot)\right),
$$

and defining block vectors $\mathbf{u}=\left[\ldots, \boldsymbol{u}^{(\alpha)}, \ldots\right]$ and similarly $\mathbf{f}$, we can write this equation with the Kronecker product as

$$
\mathbf{K u}:=\left[\sum_{\gamma} \boldsymbol{\Delta}^{(\gamma)} \otimes \mathbf{K}^{(\gamma)}\right] \mathbf{u}=\mathbf{f} .
$$

The question comes whether, and if so when, we should truncate the PCexpansion of Eq.(63), as has to be done in an actual computation. The following Theorem 3 shows that this problem is resolved when we look at the projected equation Eq.(67).

Theorem 3. The series in $\gamma$ in Eqs. $(65,67)$ is a finite sum, even when the PCexpansion Eq.(9) resp. Eq.(63) is not.

Proof. We have to look at the generic expression E $\left(H_{\beta}(\cdot) H_{\gamma}(\cdot) H_{\alpha}(\cdot)\right)$, where $\alpha, \beta \in \mathcal{J}_{M, p}$, whereas $\gamma \in \mathcal{J}$. The product $H_{\alpha}(\omega) H_{\beta}(\omega)$, as an element of the algebra of Gaussian random variables, can again be expressed as a finite sum of Hermite polynomials

$$
H_{\alpha}(\omega) H_{\beta}(\omega)=\sum_{\eta \in \mathcal{J}_{M, 2 p}} h^{(\eta)} H_{\eta}(\omega),
$$


and the index $\eta$ can only range through the finite set $\mathcal{J}_{M, 2 p}$, as the product's degree is the sum of the degrees of the individual factors. We insert this expression into a generic series in $\gamma$ :

$$
\begin{gathered}
\sum_{\gamma \in \mathcal{J}} \kappa^{(\gamma)} \mathbf{E}\left(H_{\beta}(\cdot) H_{\gamma}(\cdot) H_{\alpha}(\cdot)\right)=\sum_{\gamma \in \mathcal{J}} \kappa^{(\gamma)} \mathbf{E}\left(H_{\gamma}(\cdot) \sum_{\eta \in \mathcal{J}_{M, 2 p}} h^{(\eta)} H_{\eta}(\cdot)\right) \\
=\sum_{\gamma \in \mathcal{J}} \kappa^{(\gamma)} \sum_{\eta \in \mathcal{J}_{M, 2 p}} h^{(\eta)} \mathbf{E}\left(H_{\gamma}(\cdot) H_{\eta}(\cdot)\right) \\
=\sum_{\gamma \in \mathcal{J}} \kappa^{(\gamma)} \sum_{\eta \in \mathcal{J}_{M, 2 p}} h^{(\eta)} \delta_{\gamma \eta}=\sum_{\eta \in \mathcal{J}_{M, 2 p}} \kappa^{(\eta)} h^{(\eta)}
\end{gathered}
$$

As the set $\mathcal{J}_{M, 2 p}$ is certainly finite, this concludes the proof.

To come back to the question in this section's heading, the answer is yes when we use the Galerkin method, otherwise not directly or without other precautions, i.e. in a Monte Carlo simulation, cf. section 4.3.1.

The sum and Kronecker product structure allow savings in memory usage and coarse grain parallelisation in the numerical solution process, and we refer to the next section for references and more details on this topic.

Unfortunately, often many terms have to be used in the PC-expansion, and we would like to reduce this. One possibility is shown in the next section 4.3.3.

\subsubsection{Using the Karhunen-Loève Expansion of the Matrix}

As already stated in section 4.3.1, the direct use of the KL-expansion of the coefficient of conductivity Eq.(5), analogous to the expansion of the RHS $f(x, \omega)$ in section 3.3.3, and subsequent truncation, may lead to numerical problems. One might certainly ask why not use here also the transformation method which was advocated in section 4.3.1 for the Monte Carlo method. The appeal of the direct KL-expansion is that a similar Kronecker product structure as Eq.(67) with very few terms in the sum may be achieved, and the computation of these terms is completely analogous to the normal computation of a stiffness matrix. We show that, similarly to the result in the previous section 4.3.2 on the PC-expansion, the Galerkin projection enables the direct use of the KL-expansion without any further hypotheses on the covariance function $C_{\kappa}(x, y)$ of $\kappa(x, \omega)$.

When the space discretisation is performed for such an expansion of the conductivity, we see right away that

$$
\mathbf{K}(\omega)=\overline{\mathbf{K}}+\sum_{\jmath=1}^{\infty} \sqrt{\lambda_{\jmath}} \xi_{\jmath}(\omega) \mathbf{K}_{\jmath},
$$



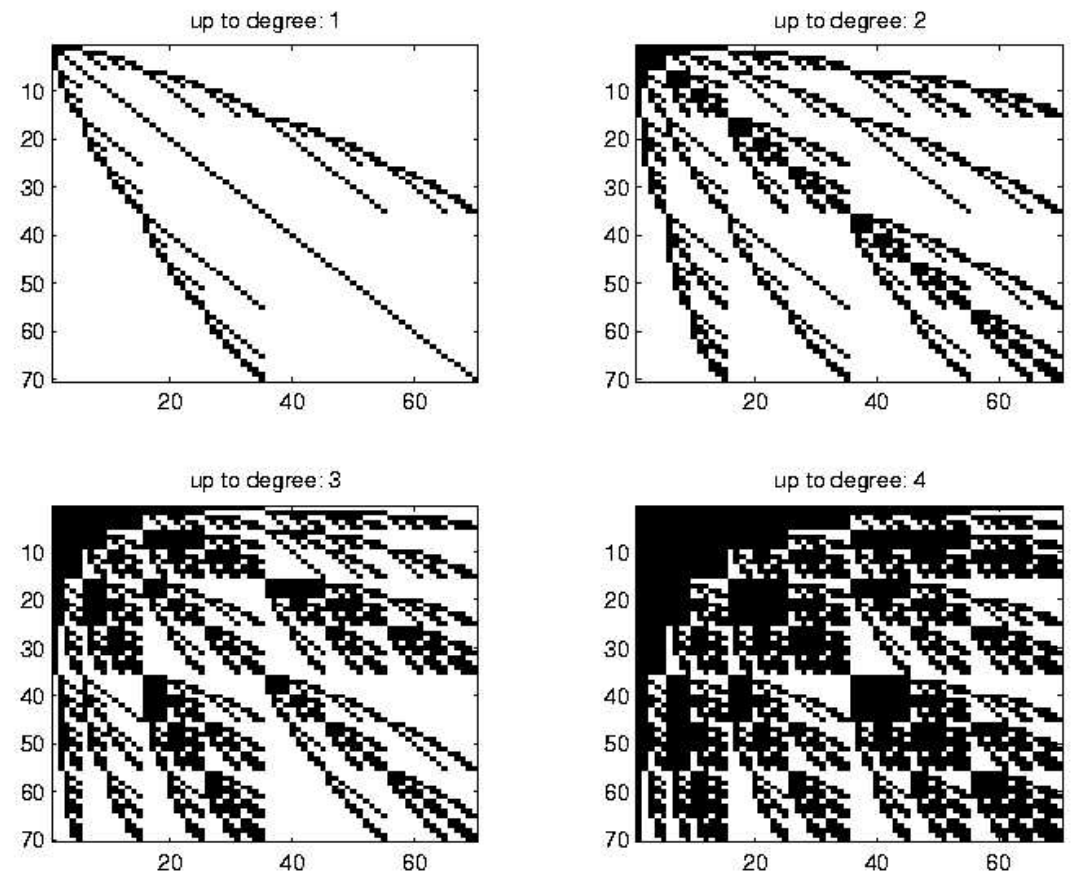

Figure 6: Sparsity Pattern of Kronecker Factor $\Delta$.

where $\mathbf{K}_{\jmath}$ is computed by using the KL-eigenfunction $\kappa_{\jmath}(x)$ as conductivity instead of $\kappa(x)$. Note that this may be usually computed with existing software, all one has to do to supply another "material", namely $\kappa_{\jmath}(x)$.

For the discrete $\boldsymbol{u}(\omega)$ we shall again use the PC-expansion as in Eq.(27) in section 3.2.2 when we were dealing with a stochastic RHS alone, or as in Eq.(64) in the preceeding section 4.3.2, and impose the Galerkin conditions to obtain the coefficients:

(70) $\forall \alpha \in \mathcal{J}_{M, p}: \quad \sum_{\beta \in \mathcal{J}_{M, p}} \mathbf{E}\left(H_{\alpha}(\cdot) \mathbf{K}(\cdot) H_{\beta}(\cdot)\right) \boldsymbol{u}^{(\beta)}=\mathbf{E}\left(H_{\alpha}(\cdot) \boldsymbol{f}\right)=\boldsymbol{f}^{(\alpha)}$ 
Expanding Eq.(70) with the series in Eq.(69) gives for all $\alpha \in \mathcal{J}_{M, p}$ :

$$
\begin{aligned}
\sum_{\beta \in \mathcal{J}_{M, p}} \mathbf{E}\left(H_{\alpha}(\cdot)\left[\overline{\mathbf{K}}+\sum_{\jmath=1}^{\infty} \sqrt{\lambda_{\jmath}} \xi_{\jmath}(\cdot) \mathbf{K}_{\jmath}\right] H_{\beta}(\cdot)\right) \boldsymbol{u}^{(\beta)} \\
=\sum_{\beta \in \mathcal{J}_{M, p}}\left[\overline{\mathbf{K}}+\sum_{\jmath=1}^{\infty} \sqrt{\lambda_{\jmath}} \mathbf{E}\left(H_{\alpha}(\cdot) \xi_{\jmath}(\cdot) H_{\beta}(\cdot)\right) \mathbf{K}_{\jmath}\right] \boldsymbol{u}^{(\beta)} \\
=\sum_{\beta \in \mathcal{J}_{M, p}}\left[\overline{\mathbf{K}}+\sum_{\jmath=1}^{\infty} \Delta_{\alpha, \beta}^{(\jmath)} \mathbf{K}_{\jmath}\right] \boldsymbol{u}^{(\beta)}=\mathbf{E}\left(H_{\alpha}(\cdot) \boldsymbol{f}\right)=\boldsymbol{f}^{(\alpha)},
\end{aligned}
$$

where we have introduced

$$
\Delta_{\alpha, \beta}^{(\jmath)}=\sqrt{\lambda_{\jmath}} \mathbf{E}\left(H_{\alpha}(\cdot) \xi_{\jmath}(\cdot) H_{\beta}(\cdot)\right) .
$$

To compute such an expectation, we again use the PC-expansion of $\xi_{\jmath}(\omega)$ Eq.(10). We know from Theorem 3 from the previous section 4.3.2, that the PC-expansion series are in this case only finite sums, i.e. the terms in Eq.(72) are finite linear combinations of terms like in Eq.(66):

$$
\mathbf{E}\left(H_{\alpha}(\cdot) \xi_{\jmath}(\cdot) H_{\beta}(\cdot)\right)=\sum_{\gamma \in \mathcal{J}_{M, 2 p}} c_{\jmath}^{(\gamma)} \mathbf{E}\left(H_{\alpha}(\cdot) H_{\gamma}(\cdot) H_{\beta}(\cdot)\right)=\sum_{\gamma \in \mathcal{J}_{M, 2 p}} c_{\jmath}^{(\gamma)} \Delta_{\alpha, \beta}^{(\gamma)} .
$$

We define similarly as in the previous section 4.3.2 the matrices $\Delta^{(\jmath)}$ with elements $\Delta_{\alpha, \beta}^{(\jmath)}$, and set $\boldsymbol{\Delta}^{(0)}=\mathbf{I}, \mathbf{K}_{0}=\overline{\mathbf{K}}$. Using again the block vectors $\mathbf{u}=\left[\ldots, \boldsymbol{u}^{(\alpha)}, \ldots\right]$ and $\mathbf{f}$, we may write this equation as

$$
\mathbf{K u}:=\left[\sum_{\jmath=0}^{\infty} \boldsymbol{\Delta}^{(\jmath)} \otimes \mathbf{K}_{\jmath}\right] \mathbf{u}=\mathbf{f}
$$

With the help of Eq.(73) we can further expand:

$$
\forall j>0: \quad \boldsymbol{\Delta}^{(\jmath)}=\sum_{\gamma \in \mathcal{J}_{M, 2 p}} \sqrt{\lambda_{\jmath}} c_{\jmath}^{(\gamma)} \boldsymbol{\Delta}^{(\gamma)},
$$

such that with Eq.(74)

$$
\mathbf{K u}=\left[\sum_{\jmath=0}^{\infty} \sum_{\gamma \in \mathcal{J}_{M, 2 p}} \sqrt{\lambda_{\jmath}} c_{\jmath}^{(\gamma)} \boldsymbol{\Delta}^{(\gamma)} \otimes \mathbf{K}_{\jmath}\right] \mathbf{u}=\mathbf{f}
$$

In Fig. 6 we see the sparsity pattern of $\Delta^{(\jmath)}$, depending on how many terms were used in the PC-expansion, produced with the MATLAB spy function. White 
space corresponds to zeros, whereas each dot represents one full spatial matrix the size of $\mathbf{K}$.

A remark similar to Remark 5 applies here:

Remark 7. There is at least one PC-term for each KL-term, hence we can expect that in an actual computation we have many less terms in Eq.(74) than in Eq.(67) —although formally there is still an infinite series in Eq.(74).

As noted in section 4.3.1, the KL-expansion converges only in $L_{2}(R)$, whereas we only have stability against perturbations in $L_{\infty}(R)$, cf. Proposition 10 . We need uniform convergence to be able to truncate the series and still guarantee the conditions Eq.(12). But the Galerkin projection helps again, as is shown in the following theorem:

Theorem 4. The series in Eq.(74) resp. Eq.(76) converges uniformly. Hence a finite number of terms suffices to keep the discrete operators $\mathbf{K}$ uniformly-in the discretisation of $V \otimes(S)$-positive definite, and therefore their inverses uniformly bounded, assuring the stability of the approximation process.

Proof. The series Eq.(74) is a series of linear operators on a finite dimensional space. The vector space of linear operators on a finite dimensional space is again finite dimensional. On a finite dimensional space all norms are equivalent, and hence the series Eq.(74) converges uniformly in the operator norm. The truncation of the series may therefore be chosen such that the discrete operators $\mathbf{K}$ are uniformly positive definite. Hence their inverses are uniformly boundedindependent of the discretisation of $V \otimes(S)$ - and consequently the approximation process is stable (cf. [68]).

Remark 8. Certainly the equivalence "constant" between different norms alluded to in Theorem 4 is dimension-dependent, and hence more terms will be needed for finer discretisations. But this will be necessary anyway in order to assure convergence and obtain the full accuracy. Again, as noted in section 2.2.1, with more requirements on the covariance function this convergence may be uniform even before the Galerkin projection (cf. also [6]). A smooth-especially around the diagonal-covariance function $C_{\kappa}(x, y)$ will cause a rapid decay of the eigenvalues $\lambda_{\jmath}$, and hence a rapid decrease of the norms of the matrices $\Delta^{(\jmath)}$ in Eq.(74). This will help the convergence, but is not necessary.

The Eq.(74) is again in tensor- or Kronecker product form, and for the computation it is definitely kept in this way [24, 57, 47, 32, 34]. Solution methods used are usually of the Krylov subspace type, where only multiplication with the system matrix $\mathbf{K}$ is necessary. In our example all the matrices $\mathbf{K}_{\jmath}$ and $\boldsymbol{\Delta}^{(\jmath)}$ are symmetric, hence so is $\mathbf{K}$. And as shown by Theorems 3 and 4, the matrices are 
also positive definite, therefore we use in our examples preconditioned conjugate gradients. There are again plenty of opportunities for coarse level parallelism, obvious in the sum and in the Kronecker product, this is described in more detail in $[32,34]$.

The PC-expansion also gives a natural multi-level structure to the equations, which can be used in the solution process $[48,49]$. An additional possibility is to select the approximating subspaces adaptively according to the functional which one wants to compute $[48,35]$.
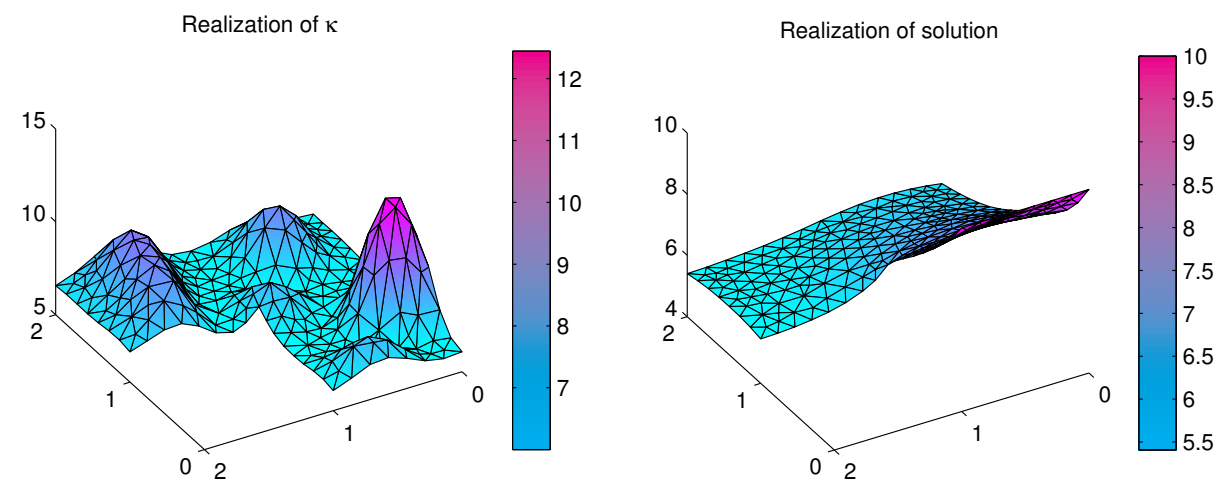

Figure 7: Realisations of Material and Solution on an L-shaped Region.

An example for a realisation for a coefficient $\kappa(x, \omega)$ and a solution $u(x, \omega)$ is shown in Fig. 7. Since these are random variables at each point, it might be more instructive to consider the pointwise mean $\bar{u}(x)$ and the pointwise variance $C_{u}(x, x)$, shown in Fig. 8
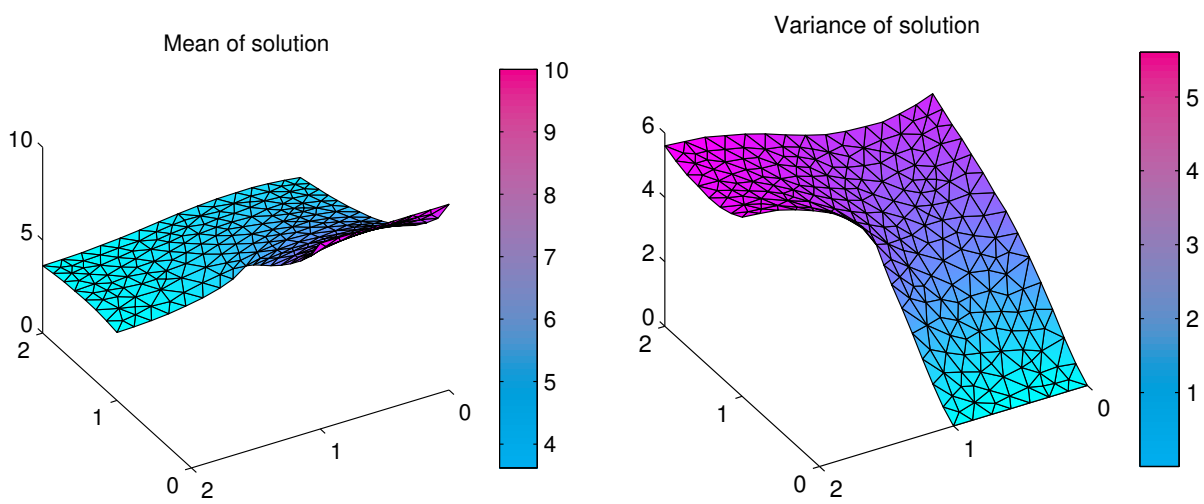

Figure 8: Mean and Variance of Solution on an L-shaped Region. 
Stochastically Independent Karhunen-Loève Expansion Terms If we have let us say two sets of coefficients $\left\{\xi_{\jmath_{1}}\right\}$ and $\left\{\xi_{\jmath_{2}}\right\}$ in the KL-expansion, which are not only uncorrelated but actually independent, then we may split

$$
\Omega=\Omega_{1} \dot{\cup} \Omega_{2} \Rightarrow L_{2}(\Omega)=L_{2}\left(\Omega_{1}\right) \oplus L_{2}\left(\Omega_{2}\right),
$$

and the first set lives only on $\Omega_{1}-\xi_{\jmath_{1}}\left(\omega_{1}\right) \in L_{2}\left(\Omega_{1}\right)$, whereas the latter are functions on $\Omega_{2}$ alone $-\xi_{\jmath_{2}}\left(\omega_{2}\right) \in L_{2}\left(\Omega_{2}\right)$.

We order the unknowns such that we have first the zero-block- $\left(\alpha_{1}, \alpha_{2}\right)=$ $(0,0)$, then those from $\Omega_{1}-\left(\alpha_{1}, \alpha_{2}\right)=(\cdot, 0)$, followed by those from $\Omega_{2}-$ $\left(\alpha_{1}, \alpha_{2}\right)=(0, \cdot)$. The ansatz basis functions are in only one of the direct summands, so that there are no cross-terms. Due to the assumption of independence, the non-zero pattern for $\mathrm{K}$ in Eq.(74) or Eq.(76) w.r.t. the $\Omega$-variables is evident from Eq.(72), and has the following bordered block-diagonal structure:

$$
\operatorname{nonzero}_{\Omega}(\mathbf{K})=\left[\begin{array}{lll}
\bullet & \bullet & \bullet \\
\bullet & \bullet & 0 \\
\bullet & 0 & \bullet
\end{array}\right]
$$

where the "•" terms are nonzero.

This means that Eqs. $(74,76)$ are systems where the variables indexed by $\alpha_{1}$ may be condensed out independently from those indexed by $\alpha_{2}$, and only in the calculation of the $(0,0)$-term - the mean-are they all added in. It is thus the assumption of independence between different KL-coefficients which generates such bordered block-diagonal systems, cf. [14, 4, 6, 7].

Stochastically Independent Source or Right Hand Side Note that if we now have a combined case with stochastic operator and stochastic RHS, all we have to do following the arguments in section 4.2 is to combine one of the approaches in this section or in section 4.3.2 with the ones from section 3. It finally means that we have to solve the problem with stochastic operator/matrix for each RHS $f^{(\alpha)}$ from the PC-expansion, or $\boldsymbol{f}_{\jmath}$ from the KL-expansion of $f(x, \omega)$.

\subsubsection{Using the Karhunen-Loève Expansion of the Solution}

The main purpose of using the KL-expansion of $\boldsymbol{u}(\omega)$ is that we hope to use it for the spatial discretisation-in the form of a post-processing model reduction, making the size of the deterministic parts $\mathbf{K}_{\jmath}$ much smaller. To represent the random part of the solution $\boldsymbol{u}(\omega)$, we know that the KL-expansion gives the best approximation in variance with the fewest number of basis vectors. So it would be good to use that here as well, like in section 3.3.4 for the additive noise case. The problem is that we know neither the mean $\overline{\boldsymbol{u}}$ nor the covariance $\mathbf{C}_{u}$ before 
we have computed the full solution $\boldsymbol{u}(\omega)$. In the previous case of only additive noise in section 3, the mean was easy to compute (one spatial solution), and the covariance and its KL-modes came out together from a generalised eigenvalue problem in section 2.2.1, independently from knowing $\boldsymbol{u}(\omega)$. In the case of a stochastic operator we have to proceed differently.

Let us first try to compute the mean in the decomposition

$$
\boldsymbol{u}(\omega)=\overline{\boldsymbol{u}}+\tilde{\boldsymbol{u}}(\omega)
$$

The equation $\mathbf{K}(\omega) \boldsymbol{u}(\omega)=\boldsymbol{f}$ shows that we can not easily average, as both $\mathbf{K}(\omega)$ and $\boldsymbol{u}(\omega)$ are correlated. Multiplying_formally_by $\mathbf{K}^{-1}(\omega)$, we see that

$$
\boldsymbol{u}(\omega)=\mathbf{K}^{-1}(\omega) \boldsymbol{f} \Rightarrow \overline{\boldsymbol{u}}=\mathbf{E}\left(\mathbf{K}^{-1}(\cdot)\right) \boldsymbol{f} .
$$

The problem is of course that it is not easy to average the inverse of a matrix. Let us split $\mathbf{K}(\omega)$ into a constant and a fluctuating part

$$
\mathbf{K}(\omega)=\hat{\mathbf{K}}+\check{\mathbf{K}}(\omega) \text {. }
$$

The simplest example of that is $\mathbf{K}(\omega)=\overline{\mathbf{K}}+\tilde{\mathbf{K}}(\omega)$, but it is not always the right one, as we want that a.e. $\left\|\hat{\mathbf{K}}^{-1} \check{\mathbf{K}}(\omega)\right\| \leq q<1$ for the Neumann series to follow. Such a split is always possible, as $\|\mathbf{K}(\omega)\|$ is bounded above and $\mathbf{K}(\omega)$ is uniformly positive definite a.e. for $\omega \in \Omega$.

With this we have

$$
\mathbf{K}(\omega) \boldsymbol{u}(\omega)=(\hat{\mathbf{K}}+\check{\mathbf{K}}(\omega)) \boldsymbol{u}(\omega)=\hat{\mathbf{K}}\left(\mathbf{I}+\hat{\mathbf{K}}^{-1} \check{\mathbf{K}}(\omega)\right) \boldsymbol{u}(\omega)=\boldsymbol{f}
$$

implying, with the use of the convergent Neumann series [22, 4]

$$
\mathbf{N}(\omega):=\left(\mathbf{I}+\hat{\mathbf{K}}^{-1} \check{\mathbf{K}}(\omega)\right)^{-1}=(\mathbf{I}+\mathbf{S}(\omega))^{-1}=\sum_{\ell=0}^{\infty}(-1)^{\ell} \mathbf{S}^{\ell}(\omega),
$$

—where $\mathbf{S}(\omega):=\hat{\mathbf{K}}^{-1} \check{\mathbf{K}}(\omega)$ with $\|\mathbf{S}(\omega)\| \leq q<1$-that

$$
\boldsymbol{u}(\omega)=\mathbf{N}(\omega) \hat{\mathbf{K}}^{-1} \boldsymbol{f}=\mathbf{N}(\omega) \hat{\boldsymbol{u}}
$$

where we have set $\hat{\boldsymbol{u}}=\hat{\mathbf{K}}^{-1} \boldsymbol{f}$.

Now we may take the average or expected value in Eq.(81)

$$
\overline{\boldsymbol{u}}=\overline{\mathbf{N}} \hat{\boldsymbol{u}}:=\mathbf{E}(\mathbf{N}(\cdot)) \hat{\boldsymbol{u}}=\left(\sum_{\ell=0}^{\infty}(-1)^{\ell} \mathbf{E}\left(\mathbf{S}^{\ell}(\cdot)\right)\right) \hat{\boldsymbol{u}}
$$


with the required computation of $\mathbf{E}\left(\mathbf{S}^{\ell}(\cdot)\right)$ now feasible. The convergence of the Neumann series in Eq.(80) implies the convergence of the one in Eq.(82). As we have an explicit expression for $\boldsymbol{u}(\omega)$ in Eq.(81), we can compute the covariance $\mathbf{C}_{u}$. Here we allow also for a stochastic (independent) RHS $\boldsymbol{f}\left(\omega_{2}\right)=\overline{\boldsymbol{f}}+\tilde{\boldsymbol{f}}\left(\omega_{2}\right)$, and set $\check{\boldsymbol{u}}\left(\omega_{2}\right)=\hat{\mathbf{K}}^{-1} \tilde{\boldsymbol{f}}\left(\omega_{2}\right)$-this is the variation caused by the stochastic RHS alone if the system were deterministic, i.e. the subject of section 3 ; the mean $\overline{\boldsymbol{u}}$ is unchanged if $\tilde{\boldsymbol{f}}\left(\omega_{2}\right) \neq 0$ - and the solution then can, with a little computation, be shown to be

$$
\boldsymbol{u}\left(\omega_{1}, \omega_{2}\right)=\mathbf{N}\left(\omega_{1}\right)\left(\hat{\boldsymbol{u}}+\check{\boldsymbol{u}}\left(\omega_{2}\right)\right)
$$

The expression for the covariance involves a lengthy computation:

$$
\begin{aligned}
\mathbf{C}_{u}= & \mathbf{E}\left(\left[\boldsymbol{u}\left(\omega_{1}, \omega_{2}\right)-\overline{\boldsymbol{u}}\right] \otimes\left[\boldsymbol{u}\left(\omega_{1}, \omega_{2}\right)-\overline{\boldsymbol{u}}\right]\right) \\
= & \mathbf{E}\left(\left[\mathbf{N}\left(\omega_{1}\right) \otimes \mathbf{N}\left(\omega_{1}\right)\right]\left[\hat{\boldsymbol{u}}+\check{\boldsymbol{u}}\left(\omega_{2}\right)\right] \otimes\left[\hat{\boldsymbol{u}}+\check{\boldsymbol{u}}\left(\omega_{2}\right)\right]\right)-\overline{\boldsymbol{u}} \otimes \overline{\boldsymbol{u}} \\
= & \mathbf{E}_{1}(\mathbf{N}(\cdot) \otimes \mathbf{N}(\cdot))\left[\hat{\boldsymbol{u}} \otimes \hat{\boldsymbol{u}}+\mathbf{E}_{2}(\check{\boldsymbol{u}}(\cdot) \otimes \check{\boldsymbol{u}}(\cdot))\right]-\overline{\boldsymbol{u}} \otimes \overline{\boldsymbol{u}} \\
= & \mathbf{E}_{1}(\mathbf{N}(\cdot) \otimes \mathbf{N}(\cdot)) \hat{\boldsymbol{u}} \otimes \hat{\boldsymbol{u}}-[\overline{\mathbf{N}} \otimes \overline{\mathbf{N}}] \hat{\boldsymbol{u}} \otimes \hat{\boldsymbol{u}} \\
& \quad+\mathbf{E}_{1}(\mathbf{N}(\cdot) \otimes \mathbf{N}(\cdot))[\hat{\mathbf{K}} \otimes \hat{\mathbf{K}}]^{-1} \mathbf{C}_{f} \\
= & {\left[\mathbf{E}_{1}(\mathbf{N}(\cdot) \otimes \mathbf{N}(\cdot))-\overline{\mathbf{N}} \otimes \overline{\mathbf{N}}\right][\hat{\mathbf{K}} \otimes \hat{\mathbf{K}}]^{-1} \overline{\boldsymbol{f}} \otimes \overline{\boldsymbol{f}} } \\
& \quad+\mathbf{E}_{1}(\mathbf{N}(\cdot) \otimes \mathbf{N}(\cdot))[\hat{\mathbf{K}} \otimes \hat{\mathbf{K}}]^{-1} \mathbf{C}_{f} .
\end{aligned}
$$

In the first term on the final line we see the covariance caused just by the stochastic matrix/operator, whereas the second tells us how the covariance of the RHS $\boldsymbol{f}\left(\omega_{2}\right)$ is amplified by the operator. For the last term we already had the (possibly low rank) expression from Eq.(41) in Theorem 1 in section 3.3.4, $[\hat{\mathbf{K}} \otimes$ $\hat{\mathbf{K}}]^{-1} \mathbf{C}_{f}=\mathbf{W} \Theta^{-2} \mathbf{W}^{*}$-we now use $\hat{\mathbf{K}}$ instead of $\mathbf{K}$-so that

$$
\mathbf{C}_{u}=\mathbf{E}_{1}(\mathbf{N}(\cdot) \otimes \mathbf{N}(\cdot))\left[\hat{\boldsymbol{u}} \otimes \hat{\boldsymbol{u}}+\mathbf{W} \Theta^{-2} \mathbf{W}^{*}\right]-\overline{\boldsymbol{u}} \otimes \overline{\boldsymbol{u}}
$$

as a practicably computable expression.

Computing the expression in Eq.(85) really means solving a large system of equations, corresponding to the boundary value problem Eq.(21) in Proposition 4 posed on $R \times R$ ! We ought to be able to do better, as-especially with the low rank approximation - the expression in Eq.(85) is also of low rank, and there should be no need to compute a full matrix. We can avoid this by observing that the 
Neumann series Eq.(80) leads to a similar series expansion of

$$
\begin{aligned}
\mathbf{N}\left(\omega_{1}\right) \otimes \mathbf{N}\left(\omega_{1}\right) & =\left(\sum_{\ell=0}^{\infty}(-1)^{\ell} \mathbf{S}^{\ell}\left(\omega_{1}\right)\right) \otimes\left(\sum_{m=0}^{\infty}(-1)^{m} \mathbf{S}^{m}\left(\omega_{1}\right)\right) \\
& =\sum_{\ell, m=0}^{\infty}(-1)^{\ell+m}\left(\mathbf{S}^{\ell}\left(\omega_{1}\right) \otimes \mathbf{S}^{m}\left(\omega_{1}\right)\right) \\
& =\sum_{k=0}^{\infty}(-1)^{k}\left(\sum_{n=0}^{k} \mathbf{S}^{n}\left(\omega_{1}\right) \otimes \mathbf{S}^{k-n}\left(\omega_{1}\right)\right)
\end{aligned}
$$

Now the expected value can be taken, and the low rank of the approximation can be reflected in the computation. Details of this will be published elsewhere. From this expression for $\mathrm{C}_{u}$ we can compute the spectrum $\Lambda_{u}$ and eigenvectors $\boldsymbol{\Phi}_{u}$, and retain only those with large eigenvalues, i.e. $\hat{\boldsymbol{\Phi}}$. Taking the QRdecomposition of $[\overline{\boldsymbol{u}}, \hat{\boldsymbol{\Phi}}]$, we have good basis for a low dimensional-reducedsubspace with which to approximate $\boldsymbol{u}(\omega)=\mathbf{Q} \boldsymbol{u}_{R}(\omega)$.

This is used for each term $\boldsymbol{u}^{(\beta)}$ in Eq.(64) in section 4.3.2,

$$
\mathbf{u}=(\mathbf{I} \otimes \mathbf{Q}) \mathbf{u}_{R},
$$

where $\mathbf{u}_{R}=\left[\ldots, \boldsymbol{u}_{R}^{(\beta)}, \ldots\right]$ is a reduced block-vector.

Projecting Eq.(76) onto this subspace, we have

$$
\begin{aligned}
&(\mathbf{I} \otimes \mathbf{Q})^{*} \mathbf{K}(\mathbf{I} \otimes \mathbf{Q}) \mathbf{u}_{R} \\
&=(\mathbf{I} \otimes \mathbf{Q})^{*}\left[\sum_{\jmath=0}^{\infty} \sum_{\gamma \in \mathcal{J}_{M, 2 p}} \sqrt{\lambda_{\jmath}} c_{\jmath}^{(\gamma)} \boldsymbol{\Delta}^{(\gamma)} \otimes \mathbf{K}_{\jmath}\right](\mathbf{I} \otimes \mathbf{Q}) \mathbf{u}_{R} \\
&=\left[\sum_{\jmath=0}^{\infty} \sum_{\gamma \in \mathcal{J}_{M, 2 p}} \sqrt{\lambda_{\jmath}} c_{\jmath}^{(\gamma)} \boldsymbol{\Delta}^{(\gamma)} \otimes \mathbf{Q}^{*} \mathbf{K}_{\jmath} \mathbf{Q}\right] \mathbf{u}_{R} \\
&=(\mathbf{I} \otimes \mathbf{Q})^{*} \mathbf{f}=\mathbf{f}_{R} .
\end{aligned}
$$

This is of the same type as as Eq.(76), but the matrices $\mathbf{Q}^{*} \mathbf{K}_{\jmath} \mathbf{Q}$ can be much smaller than $\mathbf{K}_{\jmath}$, although probably not as sparse.

Remark 9. In this approach, the mean $\overline{\boldsymbol{u}}$ and covariance $\mathbf{C}_{u}$ have been part of the computational path. For problems where also the RHS is stochastic, again the system in Eq.(88) has to be used for many RHS $\boldsymbol{f}^{(\alpha)}$. 


\subsubsection{Direct or Non-Intrusive Computation-Continued}

When we want to compute the PC-coefficients directly, we may take Eq.(89) in Proposition 9 again, only remembering that now also the matrix is stochastic:

$$
\begin{aligned}
\boldsymbol{u}^{(\alpha)}=\mathbf{E}\left(\boldsymbol{u}(\cdot) H_{\alpha}\right)=\mathbf{E}\left(\mathbf{K}^{-1}(\cdot) \boldsymbol{u}(\cdot) H_{\alpha}\right) & \\
& =\int_{\Omega} \mathbf{K}^{-1}(\omega) \boldsymbol{f}(\omega) H_{\alpha}(\omega) \Gamma(d \omega) .
\end{aligned}
$$

Approximating integrals by a quadrature rule — this could be Monte Carlowe obtain

$$
\boldsymbol{u}^{(\alpha)} \approx \sum_{z=1}^{Z} w_{z} H_{\alpha}\left(\omega_{z}\right) \mathbf{K}^{-1}\left(\omega_{z}\right) \boldsymbol{f}\left(\omega_{z}\right)=\sum_{z=1}^{Z} w_{z} H_{\alpha}\left(\omega_{z}\right) \boldsymbol{u}\left(\omega_{z}\right)
$$

with integration weights $w_{z}$ which in the case of Monte Carlo are simply $1 / Z$.

Instead of one large system equations Eq.(76), we have to solve many-indeed $Z$-small ones $\mathbf{K}\left(\omega_{z}\right) \boldsymbol{u}\left(\omega_{z}\right)=\boldsymbol{f}\left(\omega_{z}\right)$ for certain realisations $\omega_{z}$. One caveat is again that here we can not use the PC- and KL-expansion directly to represent the stochastic field, as positive definiteness and boundedness will be violated cf. section 4.3.1, but instead we recommend the transformation method Eq.(3) in section 2.2.

See [36] for a study when it is advisable to use what kind of integration in this direct computation of PC-coefficients.

It is not yet clear when it is better to use the direct approach just introduced, and when to use the coupled systems of the previous sections.

\section{The Nonlinear Case}

As often, the ability to solve the linear or at least linearised problem is the "workhorse" also for nonlinear problems. We look back to section 2, and consider again our model problem Eq.(1). Now we assume that the hydraulic conductivity $\kappa(x, u)$ depends also on the hydraulic head and on soil properties which we describe by two other fields, $\hat{\kappa}(x)$ and $\check{\kappa}(x)$. We use the model

$$
\kappa(x, u)=\hat{\kappa}(x)+\check{\kappa}(x) u(x)^{2},
$$

and we assume that both fields satisfy the boundedness and positivity conditions Eq.(12). This should be seen as a first approximation to more accurately modelled nonlinear behaviour. 


\subsection{Mathematical Formulation}

We may proceed similarly as in section 4.1. We shall first quickly formulate the deterministic and then the stochastic problem [36].

As the equation is nonlinear, the right function space for the spatial part is not anymore the Sobolev space $\stackrel{\circ}{H}{ }^{1}(R)=\stackrel{\circ}{W_{2}^{1}}(R)$ —a Hilbert space, but rather a Sobolev space $V=\stackrel{\circ}{W_{p}^{1}}(R)$ with $p>2$ - Banach space. Assume again that we are at first only dealing with a deterministic problem, so that we can take this as a 'leitmotiv'. The RHS should be in the dual space of $V$, i.e. $f \in V^{*}$, which for $V=\stackrel{\circ}{W}{ }_{p}^{1}(R)$ is $V^{*}=W_{q}^{-1}(R)$, where $1 / p+1 / q=1$.

The gradient $\nabla: \quad V \rightarrow Q$-now with $Q:=L_{p}\left(R, \mathbb{R}^{d}\right)$-is continuous, which allows us to satisfy another requirement, namely that the nonlinear $\mathrm{Ne}$ micky operator

$$
\mathrm{N}: u \mapsto \mathrm{N}(u):=\left(\hat{\kappa}+\check{\kappa} u^{2}\right) \nabla u
$$

is a continuous map from $V$ into $Q^{*}=L_{q}\left(R, \mathbb{R}^{d}\right)$, and we require $p=4$ because of the type of nonlinearity.

All this makes the semi-linear (linear in $v$ ) form $[29,55]$

$$
\mathrm{a}(u, v):=\int_{R}(\nabla v(x))^{T} \cdot \mathrm{N}(u)(x) d x
$$

hemicontinuous in $u$ and continuous in $v$, and defines a hemicontinuous (nonlinear) operator A : $V \rightarrow V^{*}$ such that for all $u, v \in V$ :

$$
\mathrm{a}(u, v)=\langle\mathrm{A}(u), v\rangle_{V},
$$

where $\langle\cdot, \cdot\rangle_{V}$ is the duality pairing between $V$ and its dual $V^{*}$. The operator A is strictly monotone and coercive. With this preparation we have the following theorem $[29,55]$ :

Theorem 5. The problem to find $u \in V$, such that for all $v \in V$

$$
\mathrm{a}(u, v)=\langle\mathrm{A}(u), v\rangle_{V}=\langle f, v\rangle_{V}
$$

has a unique solution for each $f \in V^{*}$.

In the linear case this reduces to Proposition 1.

We want to extend this to the stochastic situation and allow now both $\hat{\kappa}(x, \omega)$ and $\check{\kappa}(x, \omega)$ be random fields, still satisfying the boundedness condition Eq.(12). We look for a solution in a tensor product again. To accommodate the nonlinearity, we can not any more have the Hilbert space $L_{2}(\Omega)$ for the stochastic part of the 
solution, but this has to be the Banach space $(S):=L_{p}(\Omega)$, with $p=4$ here too, due to the type of nonlinearity, with dual space $(S)^{*}=L_{q}(\Omega)$, where again $1 / p+1 / q=1$. Hence we take as solution space the tensor product $V \otimes(S)$. This tensor product is isomorphic to $L_{p}(\Omega, V)$, i.e. a space of $V$-valued random variables. It is certainly possible to use more refined and general spaces for $(S)$, as was done in [8] for the linear Hilbert space case.

As in Definition 1 in section 3.1, we here use the operator $\nabla_{\omega}$, a linear and continuous map from $V \otimes(S)$ into $Q \otimes(S)$, in the same fashion as before.

Now we are ready to define the stochastic extension of the Nemicky operator in Eq.(92):

Definition 3. The stochastic Nemicky operator $\mathbf{N}_{\omega}: V \otimes(S) \rightarrow Q^{*} \otimes(S)^{*}$ is defined for $u \in V \otimes(S)$ by

$$
\mathbf{N}_{\omega}(u(x, \omega)):=\left(\hat{\kappa}(x, \omega)+\check{\kappa}(x, \omega) u(x, \omega)^{2}\right) \nabla_{\omega} u(x, \omega) .
$$

As before, with these ingredients we obtain a semi-linear form on $V \otimes(S)$ :

$$
\mathbf{a}(u, v):=\int_{\Omega} \int_{R}\left(\nabla_{\omega} v\right)^{T} \cdot \mathbf{N}_{\omega}(u) d x \Gamma(d \omega), \quad u, v \in V \otimes(S),
$$

which defines a hemicontinuous (nonlinear) operator A from $V \otimes(S)$ into its dual, such that for all $u, v \in V \otimes(S)$ :

$$
\mathbf{a}(u, v)=\langle\langle\mathbf{A}(u), v\rangle\rangle,
$$

where $\langle\langle\cdot, \cdot\rangle\rangle$ is the duality pairing between $V \otimes(S)$ and its dual.

This allows us to formulate the nonlinear stochastic PDE in a variational form equivalent to Eq.(95): Find $u \in V \otimes(S)$ such that for all $v \in V \otimes(S)$ :

$$
\mathbf{a}(u, v)=\langle\langle\mathbf{A}(u), v\rangle\rangle=\langle\langle f, v\rangle\rangle .
$$

Theorem 6. The variational problem Eq.(99) has a unique solution for each $f \in$ $V^{*} \otimes(S)^{*}$.

Proof. The same arguments as in the deterministic case-Theorem 5 -are used here to ascertain the existence and uniqueness of a solution $u \in V \otimes(S)$, and hence do not have to be repeated-see $[29,55]$.

\subsection{Discretisation and Numerical Approximation}

Again, like in section 4.2, a standard spatial discretisation-the same finite element spaces may be used $[12,68,77]$ — like in Eq.(23) leads to a problem of the form

$$
\mathbf{A}(\omega)[\boldsymbol{u}(\omega)]=\boldsymbol{f}(\omega),
$$


where both the nonlinear operator $\mathbf{A}(\omega)[\cdot]$ and the RHS $\boldsymbol{f}(\omega)$ are random, and hence so is $\boldsymbol{u}(\omega)$. We shall again attempt to write the solution $\boldsymbol{u}(\omega)$ as a linear combination of Hermite polynomials in $\boldsymbol{u}(\omega)=\sum_{\alpha} \boldsymbol{u}^{(\alpha)} H_{\alpha}(\omega)$, and the task will be again to compute the $\boldsymbol{u}^{(\alpha)}$. The Eq.(100) may again be termed as one where we have both additive and multiplicative noise.

Due to the nonlinearity, it is of no big advantage to take into detailed account the different sources of randomness - the operator or the RHS - like in section 4.2, as the superposition principle fails in general.

As Gaussian random variables have moments of all orders, their algebra-see section 2.2.2 - is contained in all $L_{p}(\Omega)$ spaces with $1<p<\infty$ (see [28, 43]), and is dense in these spaces. Hence the stochastic discretisation and approximation can again be performed like in section 3.2.2.

\subsection{Computational Procedures}

As we are dealing with a nonlinear equation, the different approaches for linear equations are not really much different any more. A linear expansion of the right hand side is not carried through to the solution. But certainly it pays to use the KL-expansion of the random fields involved, if only to deal with as few as possible stochastic dimensions. We have not yet developed a way to obtain the KL-vectors of the solution independently beforehand, to be used for model reduction like in section 4.3.4. So the only thing really left is to use the PC-expansion of the solution.

\subsubsection{Monte Carlo Methods-Encore}

There is not much to add here, as most of the relevant descriptions and warnings were given in earlier sections 4.3.1 and 3.3.1.

We proceed like before. We generate samples $\omega_{z}$, solve the equations etc., as described already. But now these are nonlinear equations, so the system has to be solved - although independently and possibly in parallel—many times. This can be very costly, depending on what kind of integration procedure was used [36].

\subsubsection{Using the Polynomial Chaos Expansion}

The Galerkin method is obtained by inserting the stochastic ansatz for $\boldsymbol{u}(\omega)-$ Eq.(57) —into Eq.(100). In general there will be a residuum

$$
\boldsymbol{R}(\omega)[\boldsymbol{u}(\omega)]=\boldsymbol{f}(\omega)-\mathbf{A}(\omega)[\boldsymbol{u}(\omega)]
$$

which is then projected in a standard Galerkin manner onto the finite dimensional stochastic subspace $\operatorname{span}\left\{H_{\alpha} \mid \alpha \in \mathcal{J}_{M, p}\right\}$, and we require the projection to vanish. 
This results in

$$
\mathbf{r}(\mathbf{u})=\left[\ldots, \mathbf{E}\left(H_{\alpha}(\cdot) \boldsymbol{R}(\cdot)[\mathbf{u H}(\cdot)]\right), \ldots\right]=\mathbf{0},
$$

where the same block vectors- $\mathbf{u}=\left[\ldots, \boldsymbol{u}^{(\alpha)}, \ldots\right]$-as in sections $3.4,4.3 .2$ and 4.3.3 are used.

Solution Mean

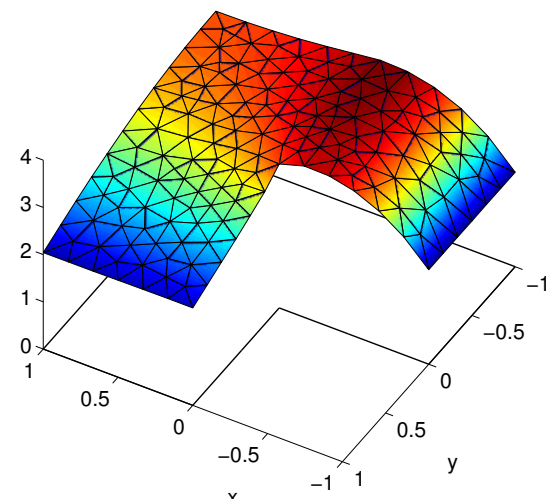

Solution Standard Deviation

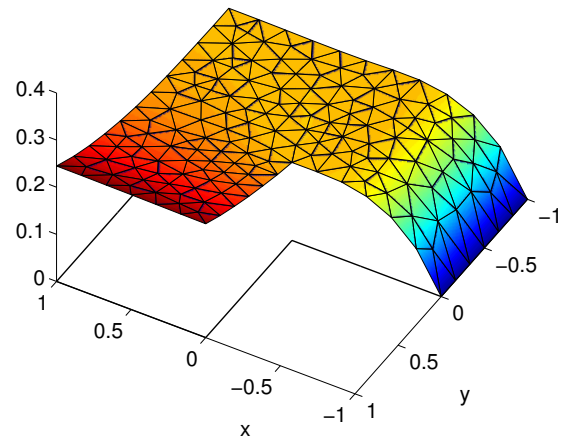

Figure 9: Mean and Standard Deviation of Solution to Nonlinear Model.

Now Eq.(102) is a huge nonlinear system, and one way to approach it is through the use of Newton's method, which involves linearisation and subsequent solution of the linearised system, employing the methods of the previous section 4.3 .

Another possibility, avoiding the costly linearisation and solution of new linear system at each iteration, is the use of Quasi-Newton methods [44, 15]. This was done in [36], and the Quasi-Newton method used-as we have a potential problem this was the BFGS-update-performed very well. The Quasi-Newton methods produce updates to the inverse of a matrix, and these low-rank changes [15] are also best kept in tensor product form [44]; so that we have tensor products here on two levels, which makes for a very economical representation.

But in any case, in each iteration the residual Eq.(102) has to be evaluated at least once, which means that for all $\left.\alpha \in \mathcal{J}_{M, p}\right\}$ the integral

$$
\mathbf{E}\left(H_{\alpha}(\cdot) \boldsymbol{R}(\cdot)[\mathbf{u H}(\cdot)]\right)=\int_{\Omega} H_{\alpha}(\omega) \boldsymbol{R}(\omega)[\mathbf{u H}(\omega)] \Gamma(d \omega)
$$

has to be computed. In general this can not be done analytically as before in the case of linear equations, and we have to resort to numerical quadrature rules-see 
section 3.3.1:

$$
\int_{\Omega} H_{\alpha}(\omega) \boldsymbol{R}(\omega)[\mathbf{u H}(\omega)] \Gamma(d \omega) \approx \sum_{z=1}^{Z} w_{z} H_{\alpha}\left(\omega_{z}\right) \boldsymbol{R}\left(\omega_{z}\right)\left[\boldsymbol{u}\left(\omega_{z}\right)\right] .
$$

What this means is that for each evaluation of the residual Eq.(102) the spatial residual Eq.(101) has to be evaluated $Z$ times-once for each $\omega_{z}$ where one has to compute $\boldsymbol{R}\left(\omega_{z}\right)\left[\boldsymbol{u}\left(\omega_{z}\right)\right]$. Certainly this can be done independently and in parallel without any communication. But we would like to point out that instead of solving the system every time for each $\omega_{z}$ as in the preceding section 5.3.1, here we only have to compute the residual-but this for every iteration.

This emphasis on integration now also points towards the direct or nonintrusive methods already mentioned in sections 3.3.5 and 4.3.5.

Error $\cdot 10^{4}$ in Mean for Galerkin

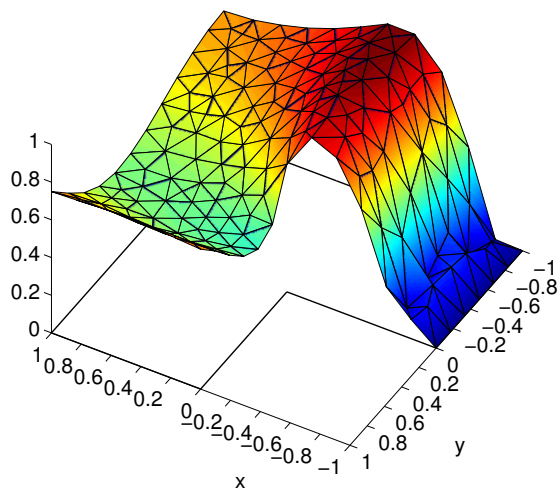

$\operatorname{Prob}\{u>3.25\}$

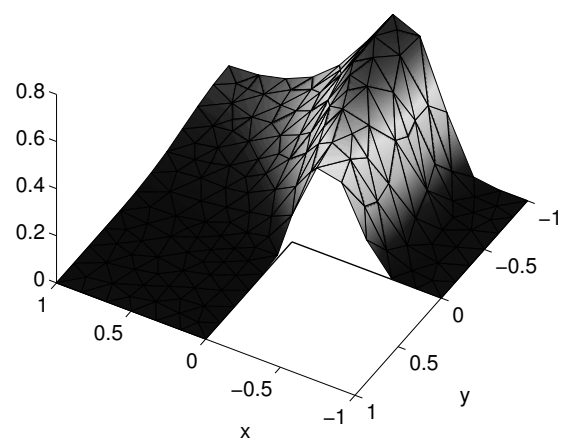

Figure 10: Error in Mean for PC-Galerkin and Example Statistic.

We compute the solution for the nonlinear groundwater flow model. The soil parameter $\kappa(x, \omega)$ is chosen beta-distributed as indicated before. As a reference, the mean and standard deviation were computed by Smolyak quadrature $S_{6}^{6}$ - to be explained in section 5.3.3 - in altogether $Z=6,188$ integration points. They are show in Fig. 9.

Next we compute the PC-expansion via the Galerkin method explained in this section, the error of which for the mean is shown in Fig. 10. We choose a polynomial chaos of degree 2 in 6 independent Gaussian variables as ansatz (28 stochastic functions). A spatial discretisation in 170 degrees of freedom was 
$u^{(\alpha)}$ for Galerkin PC-Expansion

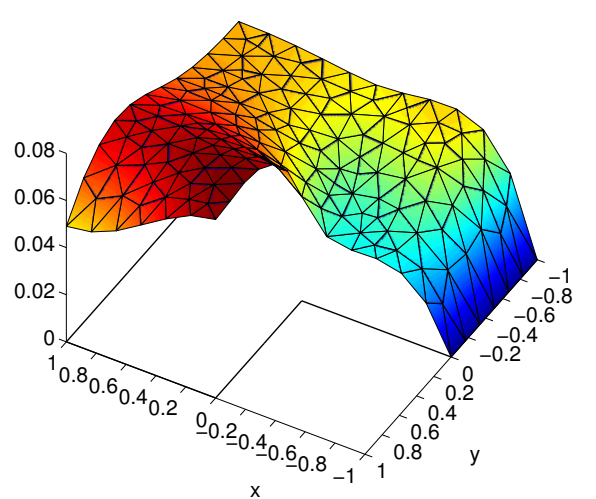

Error $\cdot 10^{4}$ in $u^{(\alpha)}$

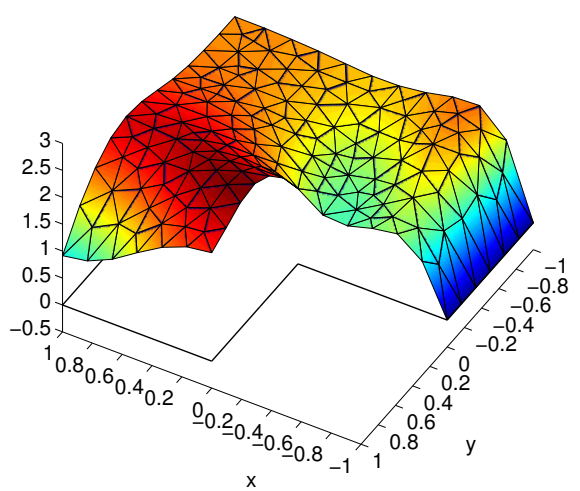

Figure 11: PC-Galerkin Vector and its Error.

performed, totalling 4,760 nonlinear equations. The BFGS solver required 19 iterations, and as the first iterations required line-searches, the residual had to be evaluated 24 times. The residual was integrated by the 5 -stage Smolyak quadrature $S_{5}^{6}$ in $Z=1,820$ integration points. As the evaluation in each integration point requires one integration in the spatial dimension, 43, 680 spatial integrations were performed.

As we now have a "response surface", we show also in Fig. 10 as an example the computation of $p_{u_{0}}(x)=\operatorname{Prob}\{u(x)>3.25\}$ for all points in the domain $R$. Next we show one of the PC-vectors in Fig. 11, and its error. It is small, at least in the "eye-ball" norm.

\subsubsection{Direct or Non-Intrusive Computation-Finale}

Like before in sections 3.3.5 and 4.3.5, we recall that the PC-coefficients of $\boldsymbol{u}(\omega)$ are also functionals- $\boldsymbol{u}^{(\alpha)}=\mathbf{E}\left(H_{\alpha}(\cdot) \boldsymbol{u}(\cdot)\right)$. When we try to evaluate this numerically, we again — as in section 5.3.1 — have to solve the nonlinear system for each integration point $\omega_{z}$; this is very similar to the MC-method. Only that we multiply by the corresponding polynomial $H_{\alpha}$ before adding everything up.

In principle this integral could be computed via $\mathrm{MC}$ integration, but now a considerable weakness of MC and QMC methods comes to the surface-they can not exploit the smoothness of the integrand. The integrand is in our case partly a polynomial and hence very smooth, but has very high variance — and that again is what the MC methods have their problem with [36]. 
We therefore focus finally on high-dimensional integration, a subject which was popping up already in several places.

\subsubsection{High-Dimensional Integration}

We only want to sketch the various possibilities, and draw some comparisons. For more in depth information we refer to the references.

Suppose that we want to evaluate the integral Eq.(103) by approximating it with a sum-a quadrature formula.

$$
\mathbf{E}(\Psi(\cdot))_{m}=\int_{\Omega_{m}} \Psi(\omega) \Gamma(d \omega) \approx \boldsymbol{\Psi}_{Z}:=\sum_{z=1}^{Z} w_{z} \Psi\left(\boldsymbol{\omega}_{z}\right),
$$

where now $m$ is the number of independent random variables, the dimension of the integration domain $\Omega_{m}$. The function $\Psi(\omega)$ is a substitute for the different integrands we have encountered so far. Several possibilities exist, and we shall briefly look at some of them.

Monte Carlo Methods Monte Carlo methods (MC-methods) - already described in section 3.3.5 - obtain the integration points as $Z$ independent random realisations of $\boldsymbol{\omega}_{z} \in \Omega_{m}$ distributed according to the probability-measure $\Gamma$ on $\Omega_{m}$, and use constant weights $w_{z}=1 / Z$. MC-methods are probabilistic as the integration points are chosen randomly, and therefore the approximation and the error are random variables. For large $Z$, the error is approximately $\|\tilde{\Psi}\|_{L_{2}} Z^{-1 / 2} \mathcal{N}(0,1)$, where $\mathcal{N}(0,1)$ is a standard-distributed Gaussian random variable, and the $L_{2}(\Omega)$-norm is the standard deviation of the zero mean fluctuating part $\tilde{\Psi}$ of the integrand.

Due to the $O\left(\|\tilde{\Psi}\|_{L_{2}} Z^{-1 / 2}\right)$ behaviour of the error, MC methods converge slowly-for instance, the error is reduced by one order of magnitude if the number of evaluations is increased by two orders. The MC methods are well suited for integrands with small variance and low accuracy requirements. In applications, their efficiency is usually increased somewhat by variance reduction and importance sampling, see e.g. $[10,63,64]$ and the references therein. The significant advantage of MC methods is that their convergence rate is independent of the dimension, in contrast with the other methods to be discussed.

Quasi-Monte Carlo Methods Quasi-Monte Carlo methods (QMC) are often seen as an alternative to Monte Carlo methods, e.g. [51, 10]. Informally speaking, they choose the sequence of integration points such that "for any number of points $Z$ the integral E (1) is approximated well by the sequence". Such sequences are called quasi-random numbers or low discrepancy sequences [51]. 
The most commonly used QMC methods have an error of $O\left(\|\tilde{\Psi}\|_{B V} Z^{-1}(\log Z)^{m}\right)$, where $\|\tilde{\Psi}\|_{B V}$ denotes the bounded variation norm. If the dimension is not too large and the integrand is smooth, the term $Z^{-1}$ dominates the error and QMC-methods may be more efficient than MC-methods, e.g. see [10] and the references therein.

Normal Quadrature Rules The textbook approach to an integral like Eq.(103) would be to take a good one-dimensional quadrature rule, and to iterate it in every dimension; this we might call the full tensor product approach.

Assume that we use one-dimensional Gauss-Hermite-formulas $Q_{k}$ with $k \in$ $\mathbb{N}$ integration points $\boldsymbol{\omega}_{\jmath, k}$ and weights $w_{\jmath, k}, \jmath=1, \ldots, k$. As is well-known, they integrate polynomials of degree less than $2 k$ exactly, and yield an error of order $O\left(k^{-(2 r-1)}\right)$ for $r$-times continuously differentiable integrands, hence takes smoothness into full account.

If we take a tensor product of these rules by iterating them $m$ times, we have

$$
\begin{aligned}
\boldsymbol{\Psi}_{Z}=Q_{k}^{m}(\Psi):=\left(Q_{k} \otimes \cdots\right. & \left.\otimes Q_{k}\right)(\Psi)=\bigotimes_{\jmath=1}^{m} Q_{k}(\Psi) \\
& =\sum_{\jmath_{1}=1}^{k} \cdots \sum_{\jmath_{m}=1}^{Z} w_{\jmath_{1}, k} \cdots w_{\jmath_{m}, k} \Psi\left(\boldsymbol{\omega}_{\jmath_{1}, k}, \ldots, \boldsymbol{\omega}_{\jmath_{m}, k}\right) .
\end{aligned}
$$

This "full" tensor-quadrature evaluates the integrand on a regular mesh of $Z=k^{m}$ points, and the approximation-error has order $O\left(Z^{-(2 r-1) / m}\right)$. Due to the exponential growth of the number of evaluation points and hence the effort with increasing dimension, the application of full tensor quadrature is impractical for high stochastic dimensions, this has been termed the "curse of dimension" [53].

Sparse or Smolyak Quadrature "Sparse" or Smolyak quadrature quadrature [67] can be applied in much higher dimensions - for recent work see e.g. [52, 54, 60] and the references therein. A software package is available at [58].

Like full tensor-quadrature, a Smolyak quadrature formula is constructed from tensor products of one-dimensional quadrature formulas, but it combines quadrature formulas of high order in only some dimensions with formulas of lower order in the other dimensions. For a multi-index $\eta \in \mathbb{N}^{m}$ with $|\eta|$ as before, the Smolyak quadrature formula is

$$
\boldsymbol{\Psi}_{Z}=S_{k}^{m}(\Psi):=\sum_{k \leq|\eta| \leq k+m-1}(-1)^{k+m-1-|\eta|}\left(\begin{array}{c}
k-1 \\
|\eta|-k
\end{array}\right) \bigotimes_{\jmath=1}^{m} Q_{\eta_{\jmath}}(\Psi) .
$$


Error $\cdot 10^{4}$ in Mean for $S_{4}^{6}$ ( $Z=455$ integration points).

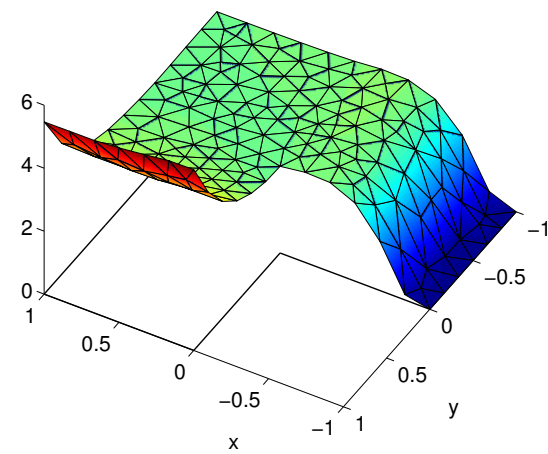

Error $\cdot 10^{3}$ in Std.-Dev. for $S_{4}^{6}(Z=455)$.

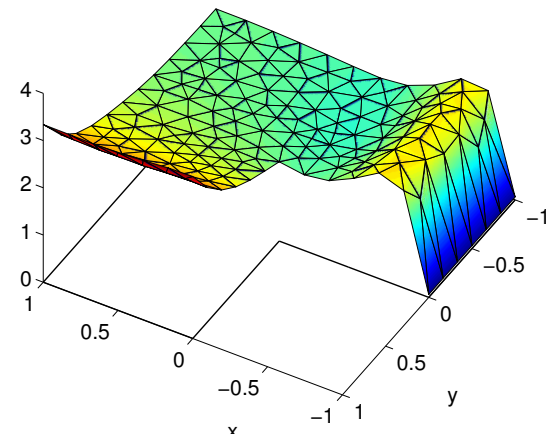

Error in Mean for Monte Carlo $(Z=500)$.

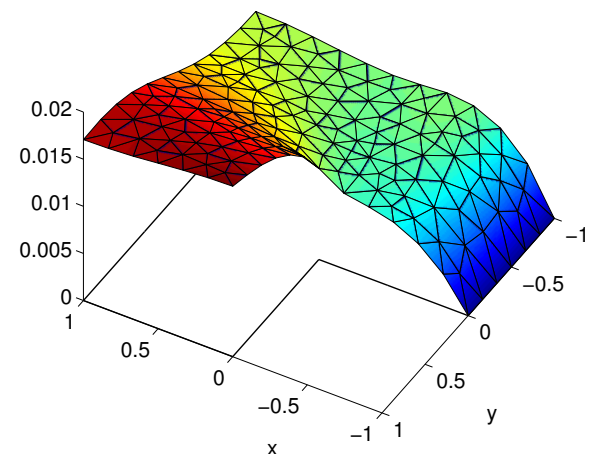

Error in Std-Dev. for Monte Carlo $(Z=500)$.

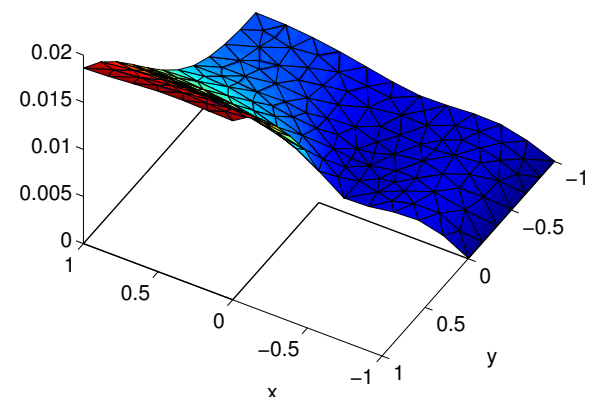

Figure 12: Solution Errors by Direct Computation 
For a fixed $k$ the number of evaluations grows significantly slower in the number of dimensions than for full quadrature. The price is a larger error: full quadrature integrates monomials $\boldsymbol{\omega}^{\eta}=\omega_{1}^{\eta_{1}} \cdots \omega_{m}^{\eta_{m}}$ exactly if their partial degree $\max _{\jmath} \eta_{\jmath}$ does not exceed $2 k-1$. Smolyak formulas $S_{k}^{m}$ integrate multivariate polynomials exactly only if their total polynomial degree $|\eta|$ is at most $2 k-1$. But still the error is only $O\left(Z^{-k}(\log Z)^{(m-1)(k+1)}\right)$ with $Z=O\left(\left[2^{k} /(k !)\right] m^{k}\right)$ evaluation points. This only grows polynomially in the dimension, and has been used up to several hundred dimensions.

Numerical Experiments We used Smolyak quadrature $S_{4}^{6}$ with 451 integration points and Monte Carlo simulation with 500 integration points. The accuracy in mean and standard deviation with respect to the reference solution are shown in Fig. 12. The errors from the naive Monte Carlo simulation are considerably larger than the error from the Smolyak integration-about forty times larger for the mean and six times larger for the standard deviation. Thus, a naive Monte Carlo simulation would require an approximately 1,600 times higher effort to obtain the same accuracy.

See [36] for some experiments, when to use which kind of integration. The finding there is that for low $m$ normal quadrature is best. For higher to moderately high (several hundred) $m$, sparse or Smolyak quadrature $[67,19,60,59,58]$ is advisable. For very high dimensions, we come into the region where first Quasi Monte Carlo [51] and then finally for extremely high dimension Monte Carlo methods should be most effective.

\section{Conclusions}

What seems to be needed-besides further development of more effective numerical algorithms - are investigations on whether and when Monte Carlo like evaluation, fully coupled Galerkin procedures, or direct PC-coefficient computation are more favourable. While the linear case seems fairly clear from the point of view of computational alternatives, it is an open problem how to obtain the mean, covariance, and KL-basis of the solution without actually computing it in the nonlinear case. Also, as the KL-basis serves as a vehicle for dimension reduction of the system, it would be interesting to see how this could be tied together with nonlinear Galerkin methods. We hope that, by exposing different computational alternatives and linking them together, we were able to shed some light on the problems involved with stationary stochastic systems. 


\section{References}

[1] R. J. Adler: The Geometry of Random Fields. John Wiley \& Sons, Chichester, 1981.

[2] K. E. Atkinson: The Numerical Solution of Integral Equations of the Second Kind. Cambridge University Press, Cambridge, 1997.

[3] I. Babuška: On randomized solutions of Laplace's equation. Casopis Pest. Mat., 1961. cited in [14].

[4] I. Babuška and P. Chatzipantelidis: On solving elliptic stochastic partial differential equations. Comp. Meth. Appl. Mech. Engrg. 191(2002)40934122.

[5] I. Babuška and J. Chleboun: Effects of uncertainties in the domain on the solution of Neumann boundary value problems in two spatial dimensions. Math. of Comp. 71(240)(2003)1339-1370.

[6] I. Babuška, R. Tempone, and G. E. Zouraris: Galerkin finite element approximations of stochastic elliptic partial differential equations. Technical report, TICAM report 02-38, Texas Institute for Computational and Applied Mathematics, University of Texas, Austin, TX, 2002. http: //www.ticam.utexas.edu/reports/0238.pdf

[7] I. Babuška, K.-M. Liu, and R. Tempone: Solving stochstic partial differential equations based on the experimental data. Math. Models \& Meth. in Appl. Sciences 13(3)(2003)415-444

[8] F. E. Benth and J. Gjerde: Convergence rates for finite element approximations for stochastic partial differential equations. Stochastics and Stochochastics Reports 63(1998)313-326.

[9] P. Besold: Solutions to Stochastic Partial Differential Equations as Elements of Tensor Product Spaces. PhD thesis, Georg-August-Universität, Göttingen, 2000.

[10] R E. Caflisch: Monte Carlo and quasi-Monte Carlo methods. Acta Numerica 7(1998)1-49.

[11] G. Christakos: Random Field Models in Earth Sciences. Academic Press, San Diego, CA, 1992.

[12] P. G. Ciarlet: The Finite Element Method for Elliptic Problems. NorthHolland, Amsterdam, 1978. 
[13] R. Courant and D. Hilbert: Methods of Mathematical Physics - Volume 1. John Wiley \& Sons, Chichester, 1989.

[14] M. K. Deb, I. Babuška, and J. T. Oden: Solution of stochastic partial differential equations using Galerkin finite element techniques. Comp. Meth. Appl. Mech. Engrg. 190(2001)6359-6372.

[15] J. E. Dennis, Jr. and R. B. Schnabel: Numerical Methods for Unconstrained Optimization and Nonlinear Equations. SIAM, Philadelphia, PA, 1996. Originally published by Prentice-Hall, Inc., Englewood Cliffs, NJ, 1983.

[16] A. Der Kuireghian and J.-B. Ke: The stochastic finite element method in structural reliability. J. Engrg. Mech. 117(12)(1988)83-91.

[17] I. Doltsinis: Inelastic deformation processes with random parameters - methods of analysis and design. Comp. Meth. Appl. Mech. Engrg. 192(2003)2405-2423.

[18] H. Elman, O. G. Ernst, D. P. O’Leary, and M. Stewart: Efficient iterative algorithms for the stochastic finite element method with application to acoustic scattering. Technical report, CS-TR \# 4423 / UMIACS TR \# 2002-102, Institute for Advanced Computer Studies, Department of Computer Science, University of Maryland, College Park, MD, 20742.

[19] T. Gerstner and M. Griebel: Numerical integration using sparse grids. $\mathrm{Nu}$ mer. Algorithms 18(1998)209-232. (also as SFB 256 preprint 553, Universität Bonn, Bonn, 1998).

[20] R. Ghanem: Ingredients for a general purpose stochastic finite elements implementation. Comp. Meth. Appl. Mech. Engrg. 168(1999)19-34.

[21] R. Ghanem: Stochastic finite elements for heterogeneous media with multiple random non-gaussian properties. ASCE J. Engrg. Mech. 125(1)(1999)24-40.

[22] R. Ghanem and P. D. Spanos: Stochastic finite elements-A spectral approach. Springer-Verlag, Berlin, 1991.

[23] D. Ghiocel and R. Ghanem: Stochastic Finite-Element Analysis of seismic soil-structure interaction. ASCE Journal Engrg. Mech. 128(1)(2002)66-77.

[24] R. Ghanem and R. Kruger: Numerical solution of spectral stochastic finite element systems. Comp. Meth. Appl. Mech. Engrg. 129(1999)289-303. 
[25] M. Griebel, P. Oswald, and T. Schiekofer: Sparse grids for boundary integral equations. Numer. Math., 83(2)(1999)279-312.

[26] A. Haldar and S. Mahadevan: Reliability assessment using stochastic finite element analysis. John Wiley \& Sons, Chichester, 2000.

[27] H. Holden, B. Øksendal, J. Ubøe, and T.-S. Zhang: Stochastic Partial Differential Equations. Birkhäuser Verlag, Basel, 1996.

[28] S. Janson: Gaussian Hilbert Spaces. Cambridge University Press, Cambridge, 1997.

[29] H. Jeggle: Nichtlineare Funktionalanalysis. BG Teubner, Stuttgart, 1979.

[30] M. Jardak, C.-H. Su, and G. E. Karniadakis: Spectral polynomial chaos solutions of the stochastic advection equation. SIAM J. Sci. Comput., 17(14)(2002)319-338.

[31] A. Keese: A review of recent developments in the numerical solution of stochastic PDEs (stochastic finite elements). Technical report, Informatikbericht 2003-6, Institute of Scientific Computing, Department of Mathematics and Computer Science, Technische Universität Braunschweig, Brunswick, June 2003.

[32] A. Keese and H. G. Matthies: Parallel solution of stochastic PDEs. Proc. Appl. Math. Mech. 2(2003)485-486.

[33] A. Keese and H. G. Matthies: Efficient solvers for nonlinear stochastic problems. In Proceedings of WCCM V-5th World Congress Comput. Mech., Vienna, 7-12 July 2002. http: / /wccm. tuwien.ac.at/

[34] A. Keese and H. G. Matthies: Parallel solution methods for stochastic systems. pp. 2023-2025 in K.-J. Bathe (ed.), Computational Fluid and Solid Mechanics - Proceedings of the 2nd MIT conference. Elsevier, Amsterdam, 2003.

[35] A. Keese and H. G. Matthies: Adaptivity and sensitivity for stochastic problems. pp. 311-316 in P. D. Spanos and G. Deodatis (eds.), Computational Stochastic Mechanics 4. Millpress, 2003.

[36] A. Keese and H. G. Matthies: Numerical methods and Smolyak quadrature for nonlinear stochastic partial differential equations. submitted to SIAM J. Sci. Comput. 
[37] M. Kleiber and T. D. Hien: The Stochastic Finite Element Method. Basic Perturbation Technique and Computer Implementation. John Wiley \& Sons, Chichester, 1992.

[38] P. E. Kloeden and E. Platen: Numerical Solution of Stochastic Differential Equations. Springer-Verlag, Berlin, 1995.

[39] A. Khuri and J. Cornell: Response Surfaces: Designs and Analyses. Marcel Dekker, New York, NY, 1987.

[40] W.-K. Liu, T. Belytschko, and A. Mani: Probabilistic finite elements for nonlinear structural dynamics. Comp. Meth. Appl. Mech. Engrg. 56(1986)6186.

[41] W.-K. Liu, T. Belytschko, and A. Mani: Random field finite elements. Int. J. Numer. Meth. Engrg. 23(10)(1986)1831-1845.

[42] M. Loève: Probability Theory. Springer-Verlag, Berlin, 1977.

[43] P. Malliavin: Stochastic Analysis. Springer-Verlag, Berlin, 1997.

[44] H. Matthies and G. Strang: The solution of nonlinear finite element equations. Int. J. Numer. Meth. Engrg. 14(1979)1613-1626.

[45] H. G. Matthies, C. E. Brenner, C. G. Bucher, and C. Guedes Soares: Uncertainties in probabilistic numerical analysis of structures and solidsstochastic finite elements. Struct. Safety, 19(3)(1997)283-336.

[46] H. G. Matthies and C. G. Bucher: Finite elements for stochastic media problems. Comp. Meth. Appl. Mech. Engrg. 168(1999)3-17.

[47] H. G. Matthies and A. Keese: Fast solvers for the white noise analysis of stochastic systems. Proc. Appl. Math. Mech. 1(2002)456-457.

[48] H. G. Matthies and A. Keese: Multilevel methods for stochastic systems. In Proc. of the 2nd Europ. Conf. Comp. Mech., ECCM-2001, Krakau, 2001

[49] H. G. Matthies and A. Keese: Multilevel solvers for the analysis of stochastic systems. pp. 1620-1622 in K.-J. Bathe (ed.), Computational Fluid and Solid Mechanics - Proceedings of the 1st MIT conference. Elsevier, Amsterdam, 2001.

[50] D. Márquez-Carreras, M. Mellouk and M. Sarrà: On stochastic partial differential equations with spatially correlated noise: smoothness of the law. Stoch. Process. Appl. 93(2001)269-284. 
[51] H. Niederreiter: Random Number Generation and Quasi-Monte Carlo Methods. SIAM, Philadelphia, PA, 1992.

[52] E. Novak and K. Ritter: High dimensional integration of smooth functions over cubes. Numer. Math. 75(1996)79-97.

[53] E. Novak and K. Ritter: The curse of dimension and a universal method for numerical integration. pp. 177-188 in G. Nürnberger, J. W. Schmidt, and G. Walz (eds.), Multivariate Approximation and Splines, ISNM. Birkhäuser Verlag, Basel, 1997.

[54] E. Novak and K. Ritter: Simple cubature formulas with high polynomial exactness. Constr. Approx. 15(1999)499-522.

[55] J. T. Oden: Qualitative Methods in Nonlinear Mechanics. Prentice-Hall, Englewood Cliffs, NJ, 1986.

[56] M. Papadrakakis and V. Papadopoulos: Robust and efficient methods for stochastic finite element analysis using Monte Carlo simulation. Сomp. Meth. Appl. Mech. Engrg. 134(1996)325-340.

[57] M. Pellissetti and R. Ghanem: Iterative solution of systems of linear equations arising in the context of stochastic finite elements. Adv. Engrg. Softw. 31(8-9)(2000)607-616.

[58] K. Petras: Smolpack - a software for Smolyak quadrature with delayed Clenshaw-Curtis basis-sequence http://www-public.tu-bs.de:8080/〜petras/software.html.

[59] K. Petras: Asymptotically minimal Smolyak Cubature, preprint, 2003.

[60] K. Petras: Fast calculation of coefficients in the Smolyak algorithm. Numer. Algorithms 26(2001)93-109.

[61] F. Riesz and B. Sz.-Nagy: Functional Analysis. Dover Publications, New York, NY, 1990.

[62] S. Sakamoto and R. Ghanem: Simulation of multi-dimensional nonGaussian non-stationary random fields Prob. Engrg. Mech. 17(2)(2002)167176.

[63] G. I. Schuëller: A state-of-the-art report on computational stochastic mechanics. Prob. Engrg. Mech., 14(4)(1997)197-321. 
[64] G. I. Schuëller: Recent developments in structural computational stochastic mechanics. pp. 281-310 in B.H.V. Topping (ed.), Computational Mechanics for the Twenty-First Century. Saxe-Coburg Publications, Edinburgh, 2000.

[65] C. Schwab and R.-A. Todor: Sparse finite elements for elliptic problems with stochastic data. Technical Report Research Report No. 2002-05, Seminar für Angewandte Mathematik, ETH Zürich, Zürich, April 2002.

[66] M. Shinozuka and G. Deodatis: Simulation of stochastic processes and fields. Prob. Engrg. Mech. 14(4)(1997)203-207.

[67] S. A. Smolyak: Quadrature and interpolation formulas for tensor products of certain classes of functions. Sov. Math. Dokl., 4(1963)240-243.

[68] G. Strang and G. J. Fix: An Analysis of the Finite Element Method. Wellesley-Cambridge Press, Wellesley, MA, 1988.

[69] B. Sudret and A. Der Kiureghian: Stochastic finite element methods and reliability. A state-of-the-art report. Technical Report UCB/SEMM-2000/08, Department of Civil \& Environmental Engineering, University of California, Berkeley, CA, 2000.

[70] T. G. Theting: Solving Wick-stochastic boundary value problems using a finite element method. Stochastics and Stochastics Reports 70(34)(2000)241-270.

[71] G. Våge: Variational methods for PDEs applied to stochastic partial differential equations. Math. Scand. 82(1)(1998)113-137

[72] E. Vanmarcke: Random Fields: Analysis and Synthesis. The MIT Press, Cambridge, MA, 3rd edition, 1988.

[73] N. Wiener: The homogeneous chaos. Amer. J. Math. 60(1938)897-936.

[74] D. Xiu and G. E. Karniadakis: The Wiener-Askey polynomial chaos for stochastic differential equations. SIAM J. Sci. Comput. 24(2)(2002)619-644.

[75] D. Xiu, D. Lucor, C.-H. Su, and G. E. Karniadakis: Stochastic modeling of flow-structure interactions using generalized polynomial chaos. ASME J. Fluid Engrg. 124(2002)51-69.

[76] D. Xiu and G. E. Karniadakis: Modeling uncertainty in steady state diffusion problems via generalized polynomial chaos. Comp. Meth. Appl. Mech. Engrg. 191(2002)4927-4948. 
[77] O. C. Zienkiewicz and R. L. Taylor: The Finite Element Method-Volume 1, the basis. Butterwort-Heinemann, Oxford, 5th ed., 2000. 
1999-04 T. Gehrke, A. Rensink

2000-01 T. Kaiser, B. Fischer, W. Struckmann

2000-02 J. Saperia, J. Schönwälder

2000-03 A. Casties

2000-04 J. Koslowski

2000-05 S. Eckstein, P. Ahlbrecht, K. Neumann

2000-06 F. Strauß, J. Schönwälder, M. Mertens

2000-07 F. Strauß

2000-08 T. Gehrke, U. Goltz

2000-09 T. Firley

2001-01 K. Diethers

2002-01 R. van Glabbeek, U. Goltz

2002-02 J. Weimar

2002-03 H. G. Matthies, M. Meyer

2002-04 H. G. Matthies, J. Steindorf

2002-05 H. G. Matthies, J. Steindorf

2002-06 H. G. Matthies, J. Steindorf

2002-07 H. Firley, U. Goltz

2003-01 M. Meyer, H. G. Matthies

2003-02 C. Täubner

2003-03 T.-P. Fries, H. G. Matthies

2003-04 A. Keese, H. G. Matthies

2003-05 A. Keese, H. G. Matthies

2003-06 A. Keese

2003-07 M. Meyer, H. G. Matthies

2003-08 H. G. Matthies, A. Keese
Konzeptioneller Entwurf mit der Unified Modeling Language

A Mobile Calculus with Data

The Modula-2 Proving System MOPS

Policy-Based Enhancements to the SNMP Framework Finite-Element-Interpolation der räumlichen Dichten eines Vielteilchensystems auf ungeordneten Gittern

A 2-dimensional view of the Chu-construction

Von parametrisierten Spezifikationen zu generierten Informationssystemen: ein Anwendungsbeispiel

JAX - A Java AgentX Sub-Agent Toolkit

Advantages and Disadvantages of the Script MIB Infrastructure

High-Level Sequence Charts with Data Manipulation

Regular languages as states for an abstract automaton

Tool-Based Analysis of Timed Sequence Diagrams

Well-behaved Flow Event Structures for Parallel

Composition and Action Refinement

Translations of Cellular Automata for Efficient

Simulation

Nonlinear Galerkin Methods for the Model Reduction of Nonlinear Dynamical Systems

Partitioned Strong Coupling Algorithms for Fluid-Structure-Interaction

Partitioned but Strongly Coupled Iteration Schemes for Nonlinear Fluid-Structure Interaction

Strong Coupling Methods

Property Preserving Abstraction for Software

Verification

Efficient Model Reduction in Non-linear Dynamics

Using the Karhunen-Loève Expansion and

Dual-Weighted-Residual Methods

Modellierung des Ethylen-Pathways mit

UML-Statecharts

Classification and Overview of Meshfree Methods

Fragen der numerischen Integration bei stochastischen finiten Elementen für nichtlineare Probleme

Numerical Methods and Smolyak Quadrature for Nonlinear Stochastic Partial Differential Equations

A Review of Recent Developments in the Numerical Solution of Stochastic Partial Differential Equations (Stochastic Finite Elements)

State-Space Representation of Instationary

Two-Dimensional Airfoil Aerodynamics

Galerkin Methods for Linear and Nonlinear Elliptic

Stochastic Partial Differential Equations 